\title{
Numerical Simulation of Cavitating Flows with Homogeneous Models
}

\author{
Eric Goncalves *, Regiane Fortes Patella \\ LEGI, 1025 rue de la Piscine, 38400 St Martin d'Heres cedex 9, France
}

\begin{abstract}
The simulation of cavitating flows is a challenging problem both in terms of modelling the physics and developing robust numerical methodologies. Such flows are characterized by important variations of the local Mach number and involve thermodynamic phase transition. To simulate these flows by applying homogeneous models, an appropriate equation of state (EOS) is necessary to cover all possible fluid states (pure liquid, two-phase mixture and pure vapour). Moreover, the numerical method has to handle any Mach number accurately. This paper presents a one-fluid compressible Reynolds-Averaged Navier-Stokes (RANS) solver with a preconditioning scheme. The cavitation phenomenon is modelled by two different liquid-vapour mixture EOS. The mathematical and thermodynamic properties are studied. Steady and unsteady numerical results are given for a Venturi geometry and comparisons are made with experimental data.
\end{abstract}

Key words: Cavitation, Homogeneous Model, Equation of State, Low Mach Number Preconditioning, RANS simulations

* Corresponding author.

Email address: Eric.Goncalves@inpg.fr (Eric Goncalves ). 


\section{Introduction}

The simulation and prediction of cavitating flows is of critical importance for the efficient design and performance of many engineering devices (turbomachinery, turbopumps in rocket propulsion systems, hydrofoils, fuel injectors, marine propellers, nozzles, underwater bodies, etc). In most cases, cavitation is an undesirable phenomenon, significantly degrading performance, resulting in reduced flow rates, lower pressure increases in pumps, load asymmetry, vibrations, noise and erosion. Cavitating flows in most industrial applications are turbulent and the dynamics of the interface formed involves complex interactions between the vapour and liquid phases. These interactions are not well understood in the closure region of cavities, where a distinct interface may not exist and where the flow is unsteady.

Several physical and numerical models have been developed to investigate cavitating flows and more generally two-phase flows with phase transition. Studies may be classified into two categories: the interface fitting method and the continuum modelling method. Interface methods assume there is a clear and distinct interface between the liquid and vapour, which can be determined via an iterative procedure. Its applications are limited to simpler problems where the cavity can be described as a well-defined closed volume of pure gas. On the other hand, a continuum method makes no attempt to track the liquid and vapour interface but treats the flow as two phases with an averaged mixture density, which continuously varies between the liquid and vapour extremes. These two-phase models are becoming more and more popular because they include the physics of cavitating flows. They are implemented using different approaches: the one-fluid method or homogeneous model, the two-fluid method and the hybrid method. The two-fluid approach assumes that both phases co-exist at every point in the flow field and each phase is governed by 
its own set of conservation laws. Because the exchanges of mass, momentum and energy are treated explicitly as transfer terms, these models can take into account the physical details occurring in the cavitation phenomenon such as mass exchange, thermal transfer and surface tension. However, the transfer terms have to be known; such quantities are usually very difficult to obtain. Moreover, these models involve non conservative terms, that is a numerical problem. Such models have been used for inviscid high-speed cavitating applications and two-phase Riemann problems $[41,58]$.

On the contrary, the one-fluid method treats the cavitating flows as a mixture of two fluids behaving as one. The governing equations are composed of three conservation laws written for the mixture. These models are based on the assumption of local kinematic equilibrium between phases (the local velocity is the same for both phases) and local thermodynamic equilibrium between the two components (local temperature and pressure equality between phases). Moreover, vaporization or condensation processes are assumed to be instantaneous. Then, this model cannot reproduce strong thermodynamic or kinetic non equilibrium effects but, because of its simplicity, it is often used for numerical simulations. The most difficult part of this approach is to define the thermodynamic behaviour of the mixture to close the system. Different ways have been explored:

- tabulated EOS and thermodynamics tables for liquid/vapour mixture properties $[7,12,54]$

- a mixture entropy maximization procedure with a relaxed pressure law [2],

- barotropic mixture laws (a sinusoidal law [9,11,37], a logarithmic law [27,32,42], a polynomial law [47], and a more complex law [46]),

- the computation of the temperature distribution to determine density variations with the Sanchez-Lacombe EOS for liquid [13],

- partial-mass and partial-density properties [29],

- the use of an algebraic system with saturation equations and thermodynam- 
ics tables [40].

Hybrid or reduced models are intermediate models between one-fluid and twofluid models, based on four or five equations. The four-equation model is obtained by adding a mass equation for the vapour or liquid density including a cavitation source term. This model is very popular in simulating cavitating flows $[1,14,19,23,26,43-45,57]$. The main difficulty is related to the formulation of the source term and the tunable parameters involved in the vaporization and condensation process (different formulations and sets of parameters are presented in [51]). The five-equation model is obtained from a simplification of a seven-equation two-fluid model. It is composed of four conservation laws plus a non conservative equation for the void ratio (see for example [33]). In the literature, this model has not been employed for cavitating flows.

Another important aspect of two-phase simulations concerns the numerical methods and CFD strategies. Cavitating flows are characterized by large variations in the local Mach number; indeed, the speed of sound can be several orders of magnitude higher in the liquid phase than in the two-phase mixture (supersonic regime). Then for low-speed applications, the numerical method must be able to properly simulate incompressible and compressible areas. In the literature, both incompressible and compressible solvers have been used. The first category requires an elliptic pressure-correction equation. For cavitating applications, density variations have to be included; see the following papers with SIMPLE or PISO pressure-based algorithms $[9,14,43,45,54,57]$. Numerical methods must be modified to take into account finite acoustic speeds and compressibility effects. According to [1], the method can lead to an erroneous acoustic speed in the mixture, particularly in the interface region, which may be not appropriate for unsteady simulations. From computations on axysymmetric bodies, it has been observed that the re-entrant jet can progress further upstream because of physical waves $[25,53]$. 
On the other hand, compressible codes solve hyperbolic equations with timemarching algorithms and requires an equation of state to evaluate the pressure. A well-known problem concerns the stiffness on the solution convergence when the Mach number becomes low. In this situation, the dominance of convection terms renders the system stiff and compressible solvers converge slowly. To overcome this difficulty, a preconditioned method is necessary. The physical acoustic waves are replaced by pseudo-acoustic modes that are much closer to the advective velocity, reducing the stiffness and enhancing the convergence. Different preconditioned compressible solvers have been used to simulate cavitating flows in water or cryogenic fluids $[1,10,13,23,42,46]$.

In addition to modelling cavitation, modelling turbulence can significantly influence the two-phase flow structures. Moreover, in large supersonic areas, the compressibility of turbulence has to be considered. For usual applications, three-dimensional time-dependent computations obtained with large eddy simulations (LES) or direct simulations (DNS) are not yet tractable. The Reynolds decomposition is often used with an averaged statistical processing resulting in the RANS equations for the mean flow quantities. This approach leads to a low-frequency separation between the modelled and computed scales. It is well known that these equations can be legitimately used for flows in which the time scale of the mean flow unsteadiness is much larger than the characteristic time scale of the turbulence. That is the case in most usual unsteady cavitating applications where the frequency of periodic shedding is less than $100 \mathrm{~Hz}$.

Moreover, the standard eddy-viscosity models based on the linear Boussinesq relation are known to suffer from numerous weaknesses. In particular, the over-production of eddy-viscosity reduces the development of unsteadiness and modifies the flow topology. For unsteady flows, the problem of limiting the turbulent viscosity becomes determinant to capture oscillations of 
cavitation sheets. Usually, arbitrary limiters are introduced in the turbulent viscosity calculation to obtain unsteady flow characteristics such as two-phase structure shedding and re-entrant jet (e.g. the limiter proposed by Reboud [8]).

This paper proposes a simple homogeneous formulation with two different mixture EOS: a barotropic law proposed by Delannoy and Kueny [11] and a mixture of stiffened gas. For both EOS, mathematical properties such as hyperbolicity and convexity are studied for the inviscid Euler equations. Numerical simulations are performed with an implicit preconditioned compressible RANS solver. The boundary conditions are treated with preconditioned characteristic relations. We test two transport-equation turbulence models associated with the Boussinesq assumption and calibrated for single-phase flows. The Menter Shear Stress Transport (SST) correction is used for unsteady computations. The models and numerical techniques are assessed by comparing the numerical results with experimental data on a Venturi geometry. Local analyses with void ratio and velocity profiles are proposed. Unsteady computations are also done on the same geometry: they show the capability of the solver to capture the dynamic behaviour of cavitation sheets.

In the following, we first summarize the essential elements of the governing equations, the modelling concepts and the numerical schemes, then present the steady and unsteady results.

\section{Governing equations and models}

The numerical simulations were carried out using an in-house CFD code solving the one-fluid compressible RANS system. First, we present the one-fluid formulation for Euler equations, with the associated preconditioned method and studies of mathematical and thermodynamic properties. Secondly, the complete RANS system is presented. 


\subsection{The one-fluid Euler equations}

In the homogeneous model, the phases are assumed to be in kinematic and thermodynamic equilibrium: they share the same pressure $P$, temperature $T$ and velocity $u$. The evolution of the two-phase flow can be described by Euler equations that employ the representative flow properties as unknowns like a single-phase problem.

The mixture density $\rho$ is defined by:

$$
\rho=\alpha \rho_{V}+(1-\alpha) \rho_{L}
$$

where $\rho_{L}$ and $\rho_{V}$ are respectively the liquid and vapour densities. The void ratio $\alpha$ characterizes the volume of vapour in each cell: $\alpha=1$ means that the cell is completely filled with vapour; inversely, a complete liquid cell is represented by $\alpha=0$. Liquid and vapour phases are characterized by their thermodynamic properties. On each cell, the unknowns are calculated by averaging them by the volume occupied.

In order to simplify the formulation, we use the one-dimensional equations, expressed in conservative variables $w=(\rho, \rho u, \rho E)$ :

$$
\begin{aligned}
\frac{\partial \rho}{\partial t}+\frac{\partial \rho u}{\partial x} & =0 \\
\frac{\partial(\rho u)}{\partial t}+\frac{\partial\left(\rho u^{2}+P\right)}{\partial x} & =0 \\
\frac{\partial(\rho E)}{\partial t}+\frac{\partial(\rho u H)}{\partial x} & =0
\end{aligned}
$$

where $E=e+u^{2} / 2$ denotes the total energy and $H=h+u^{2} / 2$ the total enthalpy; $e$ and $h$, respectively, the internal energy and the enthalpy, are related to the void ratio and the liquid and vapour values: 


$$
\begin{aligned}
& \rho e=\alpha \rho_{V} e_{V}+(1-\alpha) \rho_{L} e_{L} \\
& \rho h=\alpha \rho_{V} h_{V}+(1-\alpha) \rho_{L} h_{L}
\end{aligned}
$$

To close the system, an equation of state (EOS) and a thermal relation are necessary to link the pressure and the temperature to the thermodynamic variables. The difficulty with this homogeneous approach is to specify an EOS that covers all possible fluid states: pure liquid, two-phase mixture and pure gas. Different EOS are used in pure phases and in the mixture. In the present study, we propose a stiffened gas EOS for the pure phases and two formulations for the two-phase mixture: a mixture of stiffened gas and a barotropic EOS.

Moreover, mathematical and thermodynamic properties should be analyzed: the hyperbolicity of the inviscid system has to be verified and a suitable mixture EOS has to be convex (i.e. the Hessian matrix of internal energy has to be positive definite) to respect the second principle of thermodynamics and to obtain thermodynamically stable states.

\subsubsection{The pure-phase EOS - low Mach number preconditioning}

For pure phases, we use the convex stiffened gas (SG) equation of state (see $[24])$ :

$$
\begin{aligned}
P(\rho, e) & =(\gamma-1) \rho(e-q)-\gamma P_{\infty} \\
P(\rho, T) & =\rho(\gamma-1) C_{v} T-P_{\infty} \\
T(\rho, h) & =\frac{h-q}{C_{p}}
\end{aligned}
$$

where $\gamma=C_{p} / C_{v}$ is the polytropic coefficient, $C_{p}$ and $C_{v}$ are thermal capacities, $q$ the energy of the fluid at a given reference state and $P_{\infty}$ is a constant reference pressure. 
The associated speed of sound $c$, defined as $c^{2}=\left(\frac{\partial P}{\partial \rho}\right)_{s}=\frac{\frac{P}{\rho^{2}}-\left(\frac{\partial e}{\partial \rho}\right)_{P}}{\left(\frac{\partial e}{\partial P}\right)_{\rho}}$, can be easily computed:

$$
c^{2}=\gamma \frac{P+P_{\infty}}{\rho}=(\gamma-1) C_{p} T
$$

$c^{2}$ remains strictly positive (for $\gamma>1$ ). It ensures the hyperbolicity of the system and the existence of a convex mathematical entropy [18]. The eigenvalues of the system are $u$ and $\lambda_{ \pm}=u \pm c$.

For low Mach number applications, a preconditioning method is necessary. It is based on the modification of the derivative term by a premultiplication with a suitable preconditioning matrix. With the primitive variables $W=(P, u, s)$ where $s$ is the entropy, the preconditioned Euler equations can be expressed as:

$$
P_{e}^{-1} \frac{\partial W}{\partial t}+A_{e} \frac{\partial W}{\partial x}=0
$$

We use the preconditioning matrix proposed by Turkel $[17,50]$ :

$$
P_{e}=\left[\begin{array}{ccc}
\beta^{2} & 0 & 0 \\
0 & 1 & 0 \\
0 & 0 & 1
\end{array}\right]
$$$$
; \quad A_{e}=\left[\begin{array}{ccc}
u & \rho c^{2} & 0 \\
1 / \rho & u & 0 \\
0 & 0 & u
\end{array}\right]
$$

$\beta$ is a parameter on the order of the Mach number. In our study, we have chosen the form given by Choi and Merkle [6]:

$$
\beta^{2}=\min \left[\max \left(M^{2}, K M_{\infty}^{2}\right), 1\right]
$$


This form implies that there is no preconditioning used in transonic and supersonic flow regions (in the mixture). When $\beta^{2}=1$, the preconditioning matrix becomes the identity matrix and the system returns to its classical non preconditioned form. Moreover, for a very small flow velocity, $\beta^{2}$ is not allowed to be less than a given percentage of the freestream velocity, determined by the coefficient $K$. For inviscid computations, $K$ is on the order of unity.

The eigenvalues of the preconditioned system are:

$$
u \quad ; \quad \lambda_{ \pm}=\frac{1}{2}\left[u\left(1+\beta^{2}\right) \pm \sqrt{\left(\beta^{2}-1\right)^{2} u^{2}+4 \beta^{2} c^{2}}\right]
$$

For the conservative variables $w=[\rho, \rho u, \rho E]$, the corresponding form is:

$$
P_{c}^{-1} \frac{\partial w}{\partial t}+A_{c} \frac{\partial w}{\partial x}=0
$$

where the preconditioning matrix $P_{c}^{-1}=\frac{\partial w}{\partial W} P_{e}^{-1} \frac{\partial W}{\partial w}$ and $A_{c}$ is the Jacobian matrix of the convective fluxes. Expressions of $R=\frac{\partial w}{\partial W}$ and $R^{-1}=\frac{\partial W}{\partial w}$ are given in appendix A.

The preconditioned matrix can be written as $P_{c}^{-1}=I_{d}+\frac{\left(1-\beta^{2}\right)}{\beta^{2}(h-q)} \times B$ where $I_{d}$ is the identity matrix and the matrix $B$ is:

$$
B=\left[\begin{array}{ccc}
\frac{u^{2}}{2} & -u & 1 \\
\frac{u^{2}}{2} u & -u^{2} & u \\
\frac{u^{2}}{2}(H-q) & -u(H-q) & H-q
\end{array}\right]
$$

The matrix $B$ is idempotent (i.e. $B^{2}=B$ ) and the inverse matrix $P_{c}$ can be easily computed: $P_{c}=I_{d}+\frac{\left(\beta^{2}-1\right)}{(h-q)} \times B$. 
In the mixture area, it is not necessary to compute the preconditioned matrix . Indeed, in the compressible area, $\beta^{2}=1, P_{c}=I_{d}$ and the classical compressible system is integrated.

\subsubsection{The mixture of stiffened gas EOS - properties}

The first cavitation model applied in this study is based on a mixture of stiffened gas. On the basis of the stiffened gas EOS for each pure phase, an expression for the pressure and the temperature can be deduced from the thermal and mechanical equilibrium assumption. These expressions, function of the void ratio $\alpha$, are available in all possible fluid states:

$$
\begin{aligned}
P(\rho, e, \alpha) & =(\gamma(\alpha)-1) \rho(e-q(\alpha))-\gamma(\alpha) P_{\infty}(\alpha) \\
\frac{1}{\gamma(\alpha)-1} & =\frac{\alpha}{\gamma_{V}-1}+\frac{1-\alpha}{\gamma_{L}-1} \\
\rho q(\alpha) & =\alpha \rho_{V} q_{V}+(1-\alpha) \rho_{L} q_{L} \\
P_{\infty}(\alpha) & =\frac{\gamma(\alpha)-1}{\gamma(\alpha)}\left[\alpha \frac{\gamma_{V}}{\gamma_{V}-1} P_{\infty}^{V}+(1-\alpha) \frac{\gamma_{L}}{\gamma_{L}-1} P_{\infty}^{L}\right] \\
T(\rho, h) & =\frac{h_{L}-q_{L}}{C_{p_{L}}}=\frac{h_{V}-q_{V}}{C_{p_{V}}}=\frac{h-q(\alpha)}{C_{p}(\alpha)} \\
\rho C_{p}(\alpha) & =\alpha \rho_{V} C_{p_{V}}+(1-\alpha) \rho_{L} C_{p_{L}}
\end{aligned}
$$

The void ratio is computed with the internal energy of each phase at saturation:

$$
\alpha=\frac{\rho e-\rho_{L}^{s a t} e_{L}^{s a t}}{\rho_{V}^{s a t} e_{V}^{s a t}-\rho_{L}^{s a t} e_{L}^{s a t}}
$$

The speed of sound in the mixture can be expressed as a function of the enthalpy of each phase (see appendix B):

$$
\rho c^{2}=\rho\left(\frac{\partial P}{\partial \rho}\right)_{s}=(\gamma-1)\left[\frac{\rho_{V} \rho_{L}}{\left(\rho_{L}-\rho_{V}\right)}\left(h_{V}-h_{L}\right)\right]
$$


$c^{2}$ is always strictly positive and therefore the system is hyperbolic.

The convexity of internal energy is necessary to ensure thermodynamic stability and to respect the entropy growth criterion. The Hessian matrix of $e$ has to be positive definite. With the introduction of these dimensionless parameters:

$$
\lambda=\frac{\rho}{P}\left(\frac{\partial P}{\partial \rho}\right)_{s} \quad ; \quad g=\frac{P}{\rho T^{2}}\left(\frac{\partial T}{\partial s}\right)_{\rho} \quad ; \quad \Gamma=\frac{\rho}{T}\left(\frac{\partial T}{\partial \rho}\right)_{s}
$$

an equivalent condition of convexity was formulated by Perrier [36] for flows with phase transition:

$$
\lambda \geq 0 \quad ; \quad g \geq 0 \quad ; \quad \lambda g-\Gamma^{2} \geq 0
$$

The different parameters can be computed with the stiffened gas EOS (see Appendix C):

$$
\lambda=\frac{\rho c^{2}}{P}>0 \quad ; \quad g=\frac{\gamma P}{\rho C_{p} T}>0 \quad ; \quad \Gamma=\frac{\gamma}{\gamma-1} \frac{c^{2}}{C_{p} T}
$$

The first two conditions are always respected. The third one can be expressed as $c^{2} \geq \frac{(\gamma-1)^{2} C_{p} T}{\gamma}$.

With our choice of parameters for the stiffened gas EOS (see Section 4.2.1) and for the applications presented in this paper, the condition is verified.

\subsubsection{The barotropic EOS - properties}

The second model considered in the present work is based on a sinusoidal barotropic law (see Fig. 1) proposed by Delannoy et al. [11]. This law is characterized by its maximum slope $1 / c_{\min }^{2}$. The quantity $c_{\min }$ is an adjustable parameter of the model, which can be interpreted as the minimum speed of sound in the mixture. 
In the original approach, pure phases are considered as incompressible and the speed of sound is infinite in each phase. In our approach, in order to join compressible pure phases, we have introduced a constant $A$, close to 1 , to avoid infinite value of the speed of sound.

When the pressure becomes smaller than the quantity $P_{v a p}+\Delta P$ and greater than $P_{v a p}-\Delta P$, we have:

$$
P(\rho, \alpha)=P_{v a p}+\left(\frac{\rho_{L}^{s a t}-\rho_{V}^{s a t}}{2}\right) c_{m i n}^{2} \operatorname{Arcsin}(A(1-2 \alpha))
$$

where $\Delta P$ represents the pressure width of the law and, for a void ratio value of 0.5 , the pressure is equal to the saturation pressure $P_{v a p}$.

With this barotropic law, there is no coupling with the temperature and the cavitation phenomenon is assumed to be isothermal. The speed of sound can be computed easily:

$$
c^{2}=\left(\frac{\partial P}{\partial \rho}\right)_{s}=\left(\frac{\partial P}{\partial \rho}\right)_{T}=\frac{A c_{\min }^{2}}{\sqrt{1-A^{2}(1-2 \alpha)^{2}}}
$$

$c^{2}$ is always strictly positive and therefore the system is hyperbolic.

From Eqs. (8), (26) and (27), we introduce conditions to respect the continuity of the pressure and the continuity of the speed of sound between the liquid and the mixture (when $P=P_{v a p}+\Delta P$ ):

$$
\begin{aligned}
\Delta P & =\frac{\rho_{L}^{s a t}-\rho_{V}^{s a t}}{2} c_{\text {min }}^{2} \operatorname{Arcsin} A=\rho_{L}^{s a t}\left(\gamma_{L}-1\right) C_{v_{L}} T_{v a p}-P_{\infty}^{L}-P_{v a p} \\
c_{L}^{2} & =\frac{A c_{\text {min }}^{2}}{\sqrt{1-A^{2}}}
\end{aligned}
$$

These relations determine $A$ and set $c_{\min }$ for given values of saturation conditions. The continuity between the pure vapour and the mixture is not con- 
sidered because, in our applications, pure gas is never reached.

The dimensionless parameters for convexity conditions concerning this barotropic approach are:

$$
\lambda=\frac{\rho c^{2}}{P} \quad ; \quad g=0 \quad ; \quad \Gamma=0 \quad ; \quad \lambda g-\Gamma^{2}=0
$$

The quantity $\Delta P$ has to be smaller than the vaporization pressure $P_{v a p}$ in order to avoid negative pressure; then:

$$
c_{\text {min }}<\sqrt{\left(\frac{2}{\pi} \frac{2 P_{v a p}}{\rho_{L}^{s a t}-\rho_{V}^{s a t}}\right)}
$$

For example, for water in the ambient condition, the velocity $c_{\min }$ has to be less than $1.73 \mathrm{~m} / \mathrm{s}$. In this case, the parameter $\lambda$ is positive therefore the convexity of the EOS is verified.

\subsection{Reynolds-Averaged Navier-Stokes equations}

For turbulent computations, the compressible one-fluid RANS equations are used, coupled with a one- or two-equation turbulence model. For low Mach number applications, the inviscid preconditioner presented previously is used (no viscous terms are introduced). These equations can be expressed as:

$$
P_{c}^{-1} \frac{\partial w}{\partial t}+\operatorname{div}\left(F_{c}-F_{v}\right)=S
$$




$$
w=\left(\begin{array}{c}
\rho \\
\rho V \\
\rho E \\
\rho k \\
\rho \Psi
\end{array}\right) ; F_{c}=\left(\begin{array}{c}
\rho V \\
\rho V \otimes V+p \overline{\bar{I}} \\
(\rho E+p) V \\
\rho k V \\
\rho \Psi V
\end{array}\right) \quad ; \quad F_{v}=\left(\begin{array}{c}
0 \\
\left(\overline{\overline{\tau^{v}}}+\overline{\overline{\tau^{t}}}\right) \cdot V-Q^{v}-Q^{t} \\
\left(\mu+\mu_{t} / \sigma_{k}\right) \operatorname{grad} k \\
\left(\mu+\mu_{t} / \sigma_{\Psi}\right) \operatorname{grad} \Psi
\end{array}\right)
$$

where $w$ denotes the conservative variables, $F_{c}$ and $F_{v}$ the convective and viscous flux densities and $S$ the source terms, which concern only the transport equations. $k$ is the turbulent kinetic energy and $\Psi$ is a turbulent variable.

The exact expression of the eddy viscosity $\mu_{t}$ and the source terms depends on the turbulence model, as well as the constants $\sigma_{k}$ and $\sigma_{\Psi}$.

The total stress tensor $\overline{\bar{\tau}}$ is evaluated following the Stokes hypothesis, the Newtonian law and the Boussinesq assumption. The total heat flux vector $Q$ is obtained from the Fourier law with the constant Prandtl number hypothesis.

$$
\begin{aligned}
& \left.\overline{\bar{\tau}}=\overline{\overline{\tau^{v}}}+\overline{\overline{\tau^{t}}}=\left(\mu+\mu_{t}\right)\left[\frac{1}{2}\left(\operatorname{grad} V+(\operatorname{grad} V)^{t}\right)-\frac{2}{3}(\operatorname{div} V) \overline{\bar{I}}\right]+\frac{2}{3} k \overline{\bar{I}} 33\right) \\
& Q=Q^{v}+Q^{t}=-\left(\frac{\mu}{P_{r}}+\frac{\mu_{t}}{P_{r t}}\right) C_{p} \operatorname{grad} T
\end{aligned}
$$

In the pure liquid, the viscosity is determined by an exponential law and, in pure vapour, the viscosity follows the Sutherland law. The mixture viscosity is calculated as an arithmetic mean between the liquid and the vapour viscosity:

$$
\begin{aligned}
\mu_{L}(T) & =\mu_{0_{L}} \exp (B / T) \\
\mu_{V}(T) & =\mu_{0_{V}} \sqrt{\frac{T}{293}} \frac{1+T_{S} / 293}{1+T_{S} / T} \\
\mu(T, \alpha) & =\alpha \mu_{V}(T)+(1-\alpha) \mu_{L}(T)
\end{aligned}
$$


where $\mu_{0_{L}}, \mu_{0_{V}}, B$ and $T_{S}$ are constant parameters.

The mixture Prandtl number is also evaluated as an arithmetic mean between the liquid and the vapour value:

$$
P_{r}(\alpha)=\alpha P_{r_{V}}+(1-\alpha) P_{r_{L}}
$$

The turbulent Prandtl number $P_{r t}$ is set at 1 .

\subsubsection{Turbulence models and the wall law approach}

Two different turbulence models were used: the Menter $k-\omega$ SST model (KWSST) [30] and the one-equation Spalart-Allmaras model (SA) [48].

At the wall, a two-layer wall law approach is used:

$$
\begin{array}{llrl}
\bar{u}^{+}=y^{+} & \text {if } & y^{+}<11.13 \\
\bar{u}^{+}=\frac{1}{\kappa} \ln y^{+}+5.25 & \text { if } & y^{+}>11.13 \\
\bar{u}^{+}=\bar{u} / U_{\tau} & ; & y^{+}=\frac{y U_{\tau}}{\nu_{w}}
\end{array}
$$

In equation (39), $\bar{u}$ represents the van Driest [52] transformed velocity for compressible flows.

$$
\bar{u}=\int_{0}^{u} \sqrt{\frac{\rho}{\rho_{w}}} \mathrm{~d} u
$$

We assume that wall functions are similar in a two-phase flow and in a singlephase flow. For unsteady flows, the existence of a wall law is assumed to be 
valid at each instant.

With regard to the transport equations of the turbulence models, the production of $k$ is computed according to the formulation proposed by Viegas and Rubesin [55]. The value of $\omega$ in the first cell is obtained using the characteristic length scale of the Chen model [5].

For the one-equation Spalart-Allmaras model, the transported quantity is calculated using the models closure relations, the velocity profile and a mixinglength formulation for the eddy-viscosity. More details concerning the wall law approach are given in [16].

\section{$3 \quad$ Numerical methods}

The numerical simulations were carried out using an implicit CFD code solving the RANS/turbulent systems for multi-domain structured meshes. This solver is based on a cell-centered finite-volume discretization.

\subsection{Spatial discretization}

The system in integral form is written for a computational cell of volume $\Omega$ limited by a surface $\Sigma$, with an outer normal $n$. It can be expressed as:

$$
P_{c}^{-1} \frac{d}{d t} \int_{\Omega} w d \Omega+\oint_{\Sigma} F_{c} \cdot n d \Sigma-\oint_{\Sigma} F_{v} \cdot n d \Sigma=\int_{\Omega} S d \Omega
$$

Using the finite-volume technique for space discretization, a semi-discrete form of equation (41) can be written as:

$$
P_{c}^{-1} \Omega \frac{\partial w}{\partial t}+\sum_{\text {allfaces }}\left(F_{c}-F_{v}\right) \cdot n \Sigma=\Omega \hat{S}
$$


For the mean flow, the convective flux density vector on a cell face is computed with the space-centered Jameson scheme stabilized by a scalar artificial dissipation [20]. The artificial viscosity includes a second-order dissipation term $D_{2}$ and a fourth-order dissipation term $D_{4}$. The use of a preconditioning method modifies these dissipation terms:

$$
\begin{aligned}
& D_{2}(w)=P_{c}^{-1} \sum_{l=i, j, k} \delta_{l}\left(P_{c} \varepsilon_{l}^{(2)}\left|\lambda_{+}\right| \delta_{l} w\right) \\
& D_{4}(w)=P_{c}^{-1} \sum_{l=i, j, k} \delta_{l}\left(P_{c} \varepsilon_{l}^{(4)}\left|\lambda_{+}\right| \delta_{l}^{3} w\right)
\end{aligned}
$$

where, for each direction $l, \varepsilon_{l}^{(2)}=k^{(2)} \max \left(\nu_{l}, \nu_{l+1}\right)$ and $\varepsilon_{l}^{(4)}=\max \left(0, k^{(4)}-\varepsilon_{l}^{(2)}\right)$, $k^{(2)}$ and $k^{(4)}$ are tunable numerical coefficients. $\lambda_{+}$is the higher eigenvalue of the preconditioned system. $\delta_{l}$ is the difference operator and $\nu_{l}$ represents a sensor based on the pressure gradients. For two-phase flows, $\nu_{l}$ is also based on the density gradients.

The viscous terms are discretized by a second-order space-centered scheme. For the turbulence transport equations, the upwind Roe scheme [38] was used to obtain a more robust method. The second-order accuracy was obtained by introducing a flux-limited dissipation [49].

\subsection{Temporal discretization}

Time integration is achieved using a low-cost implicit method [28]. The implicit method consists in solving a system of equations arising from the linearization of a fully implicit scheme, at each time step. The main feature of this method is that the storage of the Jacobian matrix is completely eliminated, which leads to a low-storage algorithm. The viscous flux Jacobian matrices are replaced by their spectral radii. The convective fluxes are written with the Roe scheme instead of the Jameson scheme because of the dissipation term, the use of 
an inconsistent linearization having no consequences for steady computations. The Jacobian matrices, which appear from the linearization of the centered fluxes, are approximated with the numerical fluxes. The following system is obtained, for the iteration $n$ :

$$
\Delta w^{n}+\sum_{l=i, j, k}\left[\sigma_{l} \delta_{l} \mu_{l}\left(\Delta f_{l}^{n}\right)-\sigma_{l} \delta_{l}\left(\rho_{l}^{V} \Delta w^{n}\right)-\sigma_{l} \delta_{l}\left(D_{l} \delta_{l} \Delta w^{n}\right)\right]=R_{e x p l}^{n}
$$

where $\Delta w^{n}=w^{n+1}-w^{n}, \sigma_{l}=\Delta t / \Delta x_{l}$, and $R_{\text {expl }}^{n}$ represents the explicit conservative residuals. For each direction $l, \rho_{l}^{V}$ is the viscous spectral radius, $\mu_{l}$ is the averaged operator, $\delta_{l}$ is the difference operator, $f_{l}$ is the physical convective flux and $D_{l}$ the dissipation matrix. Then the numerical dissipation matrices are replaced by their spectral radii and the system becomes matrixfree.

With the preconditioned method, the dissipation matrices are modified, the system leads to:

$$
\begin{aligned}
\Delta w^{n} & +\sum_{l=i, j, k}\left[\sigma_{l} \delta_{l} \mu_{l}\left(\Delta f_{l}^{n}\right)-\sigma_{l} \delta_{l}\left(\rho_{l}^{V} \Delta w^{n}\right)-0.5 \sigma_{l} \delta_{l}\left(P_{c}^{-1} \rho\left(P_{c} A_{l}\right) \delta_{l} \Delta w^{n}\right)\right] \\
& =R_{\text {expl }}^{n}
\end{aligned}
$$

where $A_{l}$ is the Jacobian matrix of the convective flux for the direction $l$ and $\rho\left(P_{c} A_{l}\right)$ denotes the spectral radius of the matrix $P_{c} A_{l}$.

In this formulation, the preconditioned matrix $P_{c}^{-1}$ remains. By judiciously exploiting the idempotence propriety of the matrix $B$, it is possible to preserve a low-cost system where matrix operations and matrix-vector products can be easily computed [22].

The implicit time-integration procedure leads to a system that can be solved directly or iteratively. The direct inversion can be memory intensive and com- 
putationally expensive. Therefore, an implicit relaxation procedure is preferred and the point Jacobi relaxation algorithm was chosen.

For the turbulence transport equations, the diffusive flux Jacobian matrix is also replaced by its spectral radius. The source term needs special treatment [31]. Only the negative part of the source term Jacobian matrix is considered and replaced by its spectral radius. The system obtained is solved with a line-alternated Jacobi relaxation algorithm.

For unsteady computations, the dual time stepping method, proposed by Jameson [21], was used to solve the lack of numerical efficiency of the global time stepping approach. The derivative with respect to the physical time is discretized by a second-order formula. Making the scheme implicit with respect to the dual time provides fast convergence to the time-accurate solution. Between each time step, the solution is advanced in a dual time, and acceleration strategies developed for steady problems can be used to speed up the convergence in fictitious time. With respect to physical time, the derivative was initialized with a first-order formula.

The local time step $\Delta t$ is evaluated from stability conditions on a convective and a diffusive equation. The one-dimensional expression is:

$$
\Delta t=C F L \min \left[\frac{\Delta x}{\left|\lambda_{+}\right|}, \frac{\Delta x^{2}}{2 \rho^{V}}\right]
$$

where $C F L$ is a positive coefficient and $\Delta x$ is the cell size. 


\subsection{Inlet and outlet boundary conditions}

The numerical treatment of the boundary conditions is based on the use of the preconditioned characteristic relations [15]. The number of variables to impose at boundaries is given by the number of positive characteristics. The characteristic relations obtained for the preconditioned system, in two-dimensional flows, are:

$$
\begin{aligned}
-c^{2}\left(\rho^{c}-\rho^{s}\right)+\left(P^{c}-P^{s}\right) & =0 \\
V_{t}^{c}-V_{t}^{s} & =0 \\
\left(\lambda_{+}-V_{n}\right)\left(P^{c}-P^{s}\right)+\rho \beta^{2} c^{2}\left(V_{n}^{c}-V_{n}^{s}\right) & =0 \\
\left(\lambda_{-}-V_{n}\right)\left(P^{c}-P^{s}\right)+\rho \beta^{2} c^{2}\left(V_{n}^{c}-V_{n}^{s}\right) & =0
\end{aligned}
$$

The variables with superscript $c$ denote the variables to be computed at the boundary. Variables with superscript $s$ denote the variables obtained by the current numerical scheme. $V_{t}$ and $V_{n}$ are the tangential and the normal component of the mean velocity, respectively.

At inflow, four variables have to be imposed: we chose the stagnation pressure $P_{i}$, the stagnation temperature $T_{i}$, and the direction of the velocity. A Newton algorithm makes it possible to compute the pressure with the characteristic relation (51) and the conservative variables can be evaluated at the boundary. At outflow, only one variable is imposed: the static pressure was chosen. The conservative variables are computed with three characteristic relations (48)(50).

We assumed that inlet and outlet areas are in a pure liquid region. No cavitation appears in these boundaries.

\subsection{Wall boundary condition}

At the wall assumed to be adiabatic, a no-slip condition was used combined with a wall law treatment. It consists in imposing, in adjacent cells to a wall, 
the diffusive flux densities obtained from the analytical velocity profile (39).

\subsection{Turbulent variables}

Since the discretization scheme does not ensure the positivity of the turbulent conservative variables, limiters were used to avoid negative values. These limiters were set equal to the corresponding imposed boundary values in the inlet.

\section{Computational results on a Venturi geometry}

\subsection{Experimental conditions}

The Venturi type test section of the CREMHyG (Centre d'Essais de Machines Hydrauliques de Grenoble) cavitation small tunnel was sized and designed to simulate cavitating flows developing on the blades of space turbopump inducers. The Venturi is characterized by a convergence angle of $4.3^{\circ}$ and a divergence angle of $4^{\circ}$, illustrated in Fig. 2. The edge forming the throat of the Venturi is used to fix the separation point of the cavitation cavity. The geometrical data are:

Inlet section: $S_{i}=50 \times 44 \mathrm{~mm}^{2}$ (where the reference pressure is measured);

Throat section: $S_{\text {throat }}=43.7 \times 44 \mathrm{~mm}^{2}$;

Length of the test section (chord): $L_{r e f}=252 \mathrm{~mm}$.

This geometry is equipped with five probing holes to take various measurements such as the local void ratio, instantaneous local speed and wall pressure (Fig. 2). 
The selected operation point is characterized by the following physical parameters [3]:

$U_{\text {inlet }}=10.8 \mathrm{~m} / \mathrm{s}:$ the inlet velocity

$Q=0.02375 \mathrm{~m}^{3} / \mathrm{s}$ : the flow imposed in the circuit

$P_{\text {inlet }}=35000 \mathrm{~Pa}$ : the pressure in the inlet section

$\sigma_{\text {inlet }}=\frac{P_{\text {inlet }}-P_{\text {vap }}}{0.5 \rho U_{\text {inlet }}^{2}} \simeq 0.55$ : the cavitation parameter in the inlet section

$T_{r e f} \simeq 293 K:$ the reference temperature

$R e_{L_{r e f}}=\frac{U_{\text {inlet }} L_{r e f}}{\nu}=2.710^{6}:$ the Reynolds number

With these parameters, a cavity length $L$ ranging from $70 \mathrm{~mm}$ to $85 \mathrm{~mm}$ has been obtained, with a relatively stable aspect, as shown in Fig. 3.

\subsection{Computational parameters and mesh}

\subsubsection{Parameters of the stiffened gas EOS}

Many authors have used the stiffened gas EOS with different parameters applied to various applications with or without phase transition (essentially compressible flows and shock tube problems). In Table (1) several sets of values are given for liquid water (the speed of sound is given for a temperature $T=293 \mathrm{~K}$ ).

The stiffened gas model can provide a reasonable approximation of a fluid at high pressure. The parameters $\gamma$ and $P_{\infty}$ can be determined by these two relations :

$$
c^{2}=(\gamma-1) C_{p} T \quad \text { and } \quad \rho=\frac{P+P_{\infty}}{(\gamma-1) C_{v} T}
$$

For the water in the ambient condition, in order to respect the physical speed of sound $(c=1480 \mathrm{~m} / \mathrm{s})$ and the physical density $\left(\rho=1000 \mathrm{~kg} / \mathrm{m}^{3}\right)$, we find a large value of $\gamma: \gamma=2.8$, and an enormous value of $P_{\infty}: P_{\infty} \simeq 8.10^{8} \mathrm{~Pa}$. As a result of having a large speed of sound, liquid flows usually fall in the low 
Mach number flow regime and the coupling between the pressure and density fields weakens. That is, the variation in density is insignificant even when a very large pressure gradient is imposed on the flow, making the fluid essentially incompressible. On the other hand, small changes in the density field can result in huge changes in pressure, making numerical solutions prone to oscillations, caused by to the presence of an enormous $P_{\infty}$ value in the EOS [4].

To avoid numerical pressure oscillations, we have chosen a smaller value of $\gamma$ for the liquid: $\gamma=1.01$, because the physical ratio $C_{p} / C_{v}$ is very close to 1 . This choice allows a smaller value of $P_{\infty}$. Yet, the speed of sound is around $110 \mathrm{~m} / \mathrm{s}$ instead of $1480 \mathrm{~m} / \mathrm{s}$ in ambient conditions. When the Mach number is uniformly small, the flow is almost incompressible. There will not be large differences between a flow at Mach $=0.1$ and a flow at Mach $=0.001$. Therefore, it seems reasonable to set an artificial speed of sound in the liquid.

For the vapour, physical values were chosen, since the pure vapour was not reached in this geometry. In the mixture area, the speed of sound is computed with relation (22), which does not depend on the liquid speed of sound.

The different parameters are presented in Table (2).

\subsubsection{Parameters of the barotropic EOS}

For pure phases, the stiffened gas EOS is used with the same parameters defined on the previous section. In the mixture area, the different parameters are presented in Table (3). We tested two values for the minimum speed of sound $c_{\text {min }}$ to study the influence of this parameter. Following the works of Pascarella et al. [35] on hydrofoil geometry, this parameter has a great influence on unsteady computations: for values around $3 \mathrm{~m} / \mathrm{s}$, quasi-steady sheet cavitation was simulated, and for values around $0.8 \mathrm{~m} / \mathrm{s}$, unsteady sheet cavitation was computed. 


\subsubsection{The speed of sound in the mixture}

Figure 4 compares the evolution of the speed of sound in the mixture as a function of the void ratio for the EOS presented and the Wallis formulation [56]. The Wallis speed of sound is calculated with the assumption of local thermodynamic equilibrium and by neglecting the exchange of mass between

the two phases. The formulation is expressed as a weighted harmonic mean of the speed of sound in each phase:

$$
\frac{1}{\rho c^{2}}=\frac{\alpha}{\rho_{V} c_{V}^{2}}+\frac{1-\alpha}{\rho_{L} c_{L}^{2}}
$$

To compare the different speeds, the temperature is assumed to be constant equal to $293 \mathrm{~K}$ and saturation values are used for $\rho_{L}, \rho_{V}, h_{L}$ and $h_{V}$. For the barotropic law, $c_{m i n}$ is set at 0.857 .

We can observe that the mixture sound of speed with both EOS is smaller than the Wallis speed of sound. The speed of sound obtained with the stiffened gas EOS is not symmetric and the minimum value (around $0.65 \mathrm{~m} / \mathrm{s}$ ) is reached for a void ratio close to zero.

\subsubsection{Mesh}

The grid is a H-type topology. It contains 251 nodes in the flow direction and 62 in the orthogonal direction. A special contraction of the mesh is applied in the main flow direction just after the throat to better simulate the two-phase flow area (Fig. 5). The $y^{+}$values of the mesh, at the center of the first cell, vary between 12 and 27 for a non cavitating computation.

According to the study of mesh dependence led by Coutier [10], this grid size is adequate to simulate the steady sheet cavitation in the Venturi type section. 


\subsubsection{Numerical parameters}

For the non cavitating regime, computations are started from an uniform flowfield using a local time step. The reference temperature is set at $293 \mathrm{~K}$. The numerical parameters used are:

- the CFL number, 10

- the preconditioned parameter, $K=1$

- Jacobi iterations for the implicit stage, 15

- the two coefficients of the artificial dissipation: $k^{(2)}=0$ and $k^{(4)}=0.032$

- the farfield value of turbulent viscosity $\mu_{t_{\infty}}=0.1 \mu_{\infty}$

- the farfield value of turbulent kinetic energy $k_{\infty}=0.0045 \mathrm{~m}^{2} / \mathrm{s}^{2}$

- the farfield value of specific dissipation $\omega_{\infty}=45000 \mathrm{~s}^{-1}$

For the steady cavitating regime, computations are started from the non cavitating numerical solution. The numerical parameters are:

- the vaporization pressure, $P_{\text {vap }}=2339 P a$

- the CFL number, 0.5

- the preconditioned parameter, $K=3$

- Jacobi iterations for the implicit stage, 15

- the two coefficients of the artificial dissipation: $k^{(2)}=0.5$ and $k^{(4)}=0.032$.

For the unsteady cavitating regime, computations are performed with the dual time stepping method and are started from the non cavitating numerical solution. The numerical parameters are:

- the dimensionless time step , $\Delta t^{*}=\frac{\Delta t U_{\text {inlet }}}{L_{\text {ref }}}=0.0195$

- sub-iterations of the dual time stepping method, 100

- the CFL number, 0.5

- the preconditioned parameter, $K=3$

- Jacobi iterations for the implicit stage, 15 
- the two coefficients of the artificial dissipation: $k^{(2)}=0.5$ and $k^{(4)}=0.032$.

\subsection{Steady computations}

The experimental visualizations showed quite stable cavity behaviour for this geometry. A stable or quasi-stable cavity is characterized by a length that is almost constant in time, although the closure region always fluctuates with the existence of a re-entrant jet and little vapour cloud shedding. The reentrant jet is mainly composed of liquid, which flows upstream along the solid surface. For this geometry, no periodic cycles with large shedding have been observed. Consequently, the first analyses performed were based on steady computations.

\section{Global analyses}

Different calculations were done by considering different EOS, turbulence models, parameters and $\sigma$ values, summarized in Table (4). The goal was to obtain a stable cavitation sheet with a length close to $80 \mathrm{~mm}$. The defined sheet length used for representing the computational results was determined by the length of the iso-line corresponding to a void ratio $\alpha=0.2$.

The table shows a great sensitivity to the inlet cavitation number: a small variation of this value can generate a large discrepancy on the cavity sheet length, especially with the barotropic EOS (between cases 7 and 8, a variation of $\sigma_{\text {inlet }}<0.005$ leads to a variation of the sheet length higher than 30 $\mathrm{mm})$. Moreover, the numerical value of the inlet cavitation number is always over-predicted in comparison with the experimental value close to 0.55 , corresponding to a cavity length of about $80 \mathrm{~mm}$. When this value is decreased by modifying the outlet static pressure in boundary conditions, computations 
become unstable and do not converge, except for those using the SpalartAllmaras turbulence model and the mixture of stiffened gas EOS (case 3). In the following, six calculations were compared to experimental data: cases $2,5,7,10,12$ and 14 .

\section{Local analyses}

Complementary analyses concern local void ratio and velocity profile comparisons inside the cavity. The experimental void ratio and velocity profiles are obtained for five stations by a double optical probe (Fig. 3). The velocity is evaluated as the most probable value and the void ratio is obtained with a post-processing algorithm from the signal of the double optical probe. The relative uncertainty on the void ratio measurement was estimated at roughly $15 \%[3]$.

Figure 6 shows the evolution of the longitudinal velocity for the experiments and the six computations capturing an $80 \mathrm{~mm}$ sheet cavitation length. The overall agreement seems good between the experimental data and the simulations. This is especially true for stations 1 and 2 where no re-entrant jet phenomena occur. Further downstream, for stations 3, 4 and 5, experimental observation indicates a recirculating behaviour with a re-entrant jet extending roughly half the sheet thickness. According to experiments, this flow configuration is smoothly time fluctuating. The steady computations are not able to reproduce this situation. At the last station, with the mixture of stiffened gas EOS (SG), the presence of a recirculating area can be observed in the closure region of the cavitation sheet. This recirculation does not appear in the computations with the barotropic model.

Figure 7 illustrates experimental and numerical results concerning the void ratio. For the first station, close to the throat, the vaporization phenomenon is clearly represented. This is a relatively strong effect, and the void ratio value 
is almost equal to 0.9 near the wall. Computations with the SG mixture EOS under-estimate the maximum value of the void ratio: the discrepancy with the experimental value reaches $10 \%$. The numerical cavity thickness is slightly over-estimated. On the contrary, computations with the barotropic EOS overpredict the maximum value of the void ratio (around 8\%). By reducing the minimum speed velocity $c_{m i n}$, the maximum void ratio is decreased, as is the cavity thickness. For both EOS computations, the turbulence model has an insignificant influence.

Downstream, at the second station, the void ratio is higher (around 96\%). The distribution is similar to that obtained for station 1 , with an increase in the sheet thickness. Computations with the SG mixture EOS largely underestimate the maximum value of the void ratio (around 20\%), as observed for station 1 . The computations with the barotropic model are in very good agreement with the experimental data, especially with the smallest value of $c_{\min }$. From the third station, the re-entrant jet becomes noticeable, as observed before in the velocity field analyses. As this point, the void ratio values are over-estimated by all computations. As noted above, the computations with the stiffer barotropic law predict vey well the cavity thickness.

At stations 4 and 5 , the void ratio is substantially over-estimated by numerical computations, mainly because of the unsteady character of the sheet. In the same way, the thickness of the cavitation is over-predicted by all the computations.

At the last station, oscillations of the void ratio are evidenced for all computations because of the unsteadiness of the flow. With the SG EOS, the recirculating flow, noted on the velocity profile, induces a small re-entrant jet with liquid water in the wall region up to a distance $y$ around $0.003 \mathrm{~m}$. This phenomenon is not observed on the experimental data.

The wall pressure profiles are plotted in Fig. 8 versus the distance $x-x_{\text {inlet }}$. The first five data are located inside the cavity (where the void ratio and 
velocity profiles are measured). For all computations, the pressure remains at an almost constant value in the cavity. With the SG EOS, this constant pressure is inferior to the vaporization pressure $P_{\text {vap }}$. This could be due to the stiffness of the EOS and the choice of the saturation values used in the model. Downstream, large discrepancies are notable for all computations because of the re-entrant jet. The re-compression is respectively lower and higher for calculations with barotropic and SG models in comparison with the experimental data.

These steady computations evaluate physical and numerical models better. In steady situations (stations 1 and 2), the results are in quite good agreement with the experimental data. The stiffer barotropic law shows better results on the void ratio profiles inside the sheet. For all computations, the influence of the turbulence model seems to be low. A better representation of re-entrant jet was obtained by unsteady calculations, as presented in the following section.

\subsection{Unsteady computations}

Unsteady computations were done with both EOS and the $k-\omega$ SST turbulent model. The Spalart-Allmaras model, known to over-produce the turbulent viscosity and to dampen the unsteadiness of the flow, was not used.

\section{Global analyses}

Different calculations were done by considering different $\sigma_{\text {inlet }}$ values, summarized in Table (5). The goal was to obtain a quasi-stable cavitation sheet whose length varied between $70-85 \mathrm{~mm}$ and a re-entrant jet.

First of all, three types of behaviour can be distinguished for the cavity by 
decreasing the $\sigma_{\text {inlet }}$ values. Figure 9 illustrates the different cavities where the time-averaged void ratio is plotted.

a) an attached cavity sheet with a small recirculation area at the closure of the sheet (cases 2, 3 and 8).

b) an attached cavity sheet with a large re-entrant jet and with the presence of small clouds of mixture in the closure region of the sheet (cases 4, 5, 6, 9 and 10). This configuration of quasi-stable sheet is in good agreement with the experimental data.

c) an attached cavity sheet with large two-phase structures (cases 7 and 11). The re-entrant jet led to the break-off of the cavity, generating a large structure with high void ratio value $(\alpha=90 \%)$. In our computations, this structure is not convected or dissipated in the flow.

Secondly, calculations obtained with the mixture of stiffened gas EOS (SG) are not able to capture a quasi-stable sheet with a re-entrant jet. As observed in the steady computations, the numerical value of the inlet cavitation number is always over-predicted in comparison with the experimental value, close to 0.55 , corresponding to $L_{\text {cav }} \simeq 80 \mathrm{~mm}$.

In the following, four simulations were compared to experimental data: cases 5, 6, 9 and 10, which predict a quasi-stable cavity sheet with a significant re-entrant jet. The cavities obtained are presented in Fig. 10, where the timeaveraged void ratio for each computation is plotted. The attached cavity sheet can clearly be seen just behind the throat and clouds of mixture in the sheet's closure region. 


\section{Local analyses}

Figure 11 illustrates the evolution of the longitudinal velocity for the experiments and the numerical results. The numerical value is obtained by a timeaveraged treatment.

At stations 1 and 2, a good agreement is observed and all computations give similar results. At stations 3, 4 and 5, the recirculating behaviour with a reentrant jet is simulated by all computations. However, the thickness of this recirculating area is largely under-estimated by all calculations. For the last station, the intensity of the recirculating zone near the wall is under-estimated by all calculations.

Figure 12 shows the experimental and numerical results concerning the void ratio. The numerical values were obtained by a time-averaged treatment.

For the first and second station, close to the Venturi throat, the results obtained by computations are in good agreement with the experimental data, predicting the cavity thickness very well.

At station 3, the numerical results are sensitive to the value of the minimum speed of sound $c_{\text {min }}$. With the highest value of this parameter, the maximum value of the void ratio is over-estimated (around 30\%). Moreover, a liquid reentrant jet $(\alpha=0)$ can be seen close to the wall, up to a distance $y=0.001$ m. On the other hand, the re-entrant jet captured by the calculations with the stiffer barotropic law is not composed of pure liquid. At the wall, the void ratio value is around 0.28 for case $9(\sigma=0.626)$, which is in very good agreement with the experimental value equal to 0.274 . For case $10(\sigma=0.608)$, the wall value is around 0.36 . Moreover, above the wall, from $y=0.001$ to $y=0.003$, a pure liquid area is evidenced by computations, which is not observed in the time-averaged void ratio measurements. The maximum void ratio value is also better predicted by the calculations with the smaller $c_{\min }$ value. For cases 9 and 10, the error is respectively around $10 \%$ and $16 \%$. From stations 3 to 5 , all the computations considered simulated a large re-entrant jet with the 
presence of mixture clouds and a pure liquid area (re-compression zone that can be seen in the wall pressure distribution in Fig. 13).

At station 4, the void ratio is greatly over-estimated by numerical computations with the greater value of $c_{\text {min }}$, and the re-entrant is no longer composed of pure liquid. For cases 5 and 6 , we observed a large cloud of mixture with large void ratio values. The behaviour is different for cases 9 and 10, with the smallest $c_{\min }$ value. For case 9, a pure liquid zone with a small cloud of mixture can be seen, whereas, for case 10, two small mixture clouds are present. Finally, at station 5, for both cases 5 and 6 , the length of the sheet is shorter than the experimental measurement and the computations predict a recirculation zone with pure liquid. For cases 9 and 10, a small mixture cloud is again present above a large pure liquid area.

The wall pressure profiles are plotted in Fig. 13 versus the distance $x-x_{\text {inlet }}$. These values are obtained by a time-averaged treatment for both experiments and calculations. Near station 3, for all computations, we can observe a peak of pressure above the vaporization pressure, indicating the presence of a pure liquid area. Yet, this peak is not visible on experimental values. Downstream, as noted with the steady calculations with the barotropic EOS, the re-compression is lower in comparison with the experimental data.

These unsteady computations showed that the mixture of stiffened gas EOS failed to predict a quasi-stable cavity sheet with a re-entrant jet. This aspect must be confirmed with other unsteady applications. Only the barotropic law provided good-quality results, both for velocity profiles and void ratio values. To model turbulence, the SST correction of Menter captured the unsteady behaviour of the flow and no arbitrary limiter was added. 


\section{Conclusion}

A two-phase one-fluid compressible CFD method was presented and applied to Venturi cavitating flows. Several numerical aspects of the method were outlined, including a low Mach number preconditioner, a low-storage implicit dual-time strategy and preconditioned characteristic relations for the boundary conditions treatment. Two different cavitation models based on two mixtures EOS were tested and compared. The convexity conditions were analyzed and verified. To model turbulence, compressible wall functions associated with the Menter $k-\omega$ SST model were used.

The numerical results presented verify the ability of the numerical tool to accurately simulate cavitating flows on a Venturi geometry. Global and local analyses of flows were proposed based on steady and unsteady calculations. Simulations demonstrated mainly the influence of the mixture EOS.

Unsteady simulations applying the mixture of stiffened gas EOS associated with the Menter k-w SST model provide a stable sheet without significant re-entrant jet and do not agree with the qualitative behaviour observed experimentally. In further work, this cavitation model should be associated with other turbulence models and should be evaluated on different unsteady applications with unstable behaviour and periodic shedding.

The barotropic model made it possible to obtain good-quality results in comparison with experimental data (void ratio and velocity profiles) by capturing a quasi-stable behaviour of the cavity sheet with re-entrant jet. In relation to previous numerical studies developed by our team on the same Venturi geometry (a similar barotropic model and two other numerical approaches $[3,9,10]$ ), it is worth noting that the present numerical study:

a) provided a better simulation of the qualitative and quantitative unsteady behaviour of the cavitating flow and a more accurate prediction of local fields; b) presented analyses of thermodynamic proprieties of the applied barotropic EOS, which provides a better evaluation of $c_{\text {min }}$, the minimum speed of sound 
in the mixture.

Additional works are in progress to simulate cavitating flows with cryogenic fluids, to develop other numerical schemes and to pursue comparative analyses between numerical approaches, turbulence and cavitation models.

\section{Appendix}

Appendix A: the preconditioned system for pure phases

For pure phases, which follow the stiffened gas EOS, the different matrices are:

$$
R=\frac{\partial W}{\partial w}=\left[\begin{array}{ccc}
\frac{(\gamma-1)}{2} u^{2} & -(\gamma-1) u & (\gamma-1) \\
-u / \rho & 1 / \rho & 0 \\
-\frac{(\gamma-1) u^{2}}{2\left(P+P_{\infty}\right)}-\frac{\gamma}{\rho} & -\frac{(\gamma-1) u}{P+P_{\infty}} & \frac{\gamma-1}{P+P_{\infty}}
\end{array}\right]
$$

$$
R^{-1}=\frac{\partial w}{\partial W}=\left[\begin{array}{ccc}
\frac{1}{c^{2}} & 0 & \frac{-\rho}{\gamma} \\
\frac{u}{c^{2}} & \rho & \frac{-\rho u}{\gamma} \\
\frac{H-q}{c^{2}} & \rho u & \frac{\rho u^{2}}{2 \gamma}
\end{array}\right]
$$


Appendix B: the speed of sound in a mixture of stiffened gas

Starting from the usual thermodynamic relation

$$
d e=T d s+\frac{P}{\rho^{2}} d \rho \quad \text { or } \quad d(\rho e)=\rho T d s+h d \rho
$$

And with the differential of $\rho e$ :

$$
d(\rho e)=\left(\frac{\partial \rho e}{\partial \rho}\right)_{P} d \rho+\left(\frac{\partial \rho e}{\partial P}\right)_{\rho} d P
$$

We can obtain the differential of the pressure $P$ :

$$
\left(\frac{\partial \rho e}{\partial P}\right)_{\rho} d P=\rho T d s+\left[h-\left(\frac{\partial \rho e}{\partial \rho}\right)_{P}\right] d \rho
$$

We deduce an expression of the speed of sound:

$$
c^{2}=\left(\frac{\partial P}{\partial \rho}\right)_{s}=\frac{h-\left(\frac{\partial \rho e}{\partial \rho}\right)_{P}}{\left(\frac{\partial \rho e}{\partial P}\right)_{\rho}}
$$

With the stiffened gas EOS, we have:

$$
\begin{aligned}
\left(\frac{\partial \rho e}{\partial P}\right)_{\rho} & =\alpha\left(\frac{\partial \rho_{V} e_{V}}{\partial P}\right)_{\rho}+(1-\alpha)\left(\frac{\partial \rho_{L} e_{L}}{\partial P}\right)_{\rho}=\frac{1}{\gamma-1} \\
\left(\frac{\partial \rho e}{\partial \rho}\right)_{P} & =\frac{\partial}{\partial \rho}\left[\alpha\left(\frac{P}{\gamma_{V}-1}+\rho_{V} q_{V}+\frac{\gamma_{V}}{\gamma_{V}-1} P_{\infty}^{V}\right)+(1-\alpha)\left(\frac{P}{\gamma_{L}-1}+\rho_{L} q_{L}+\frac{\gamma_{L}}{\gamma_{L}-1} P_{\infty}^{L}\right)\right] \\
& =\frac{\rho_{L} h_{L}-\rho_{V} h_{V}}{\rho_{L}-\rho_{V}}
\end{aligned}
$$

Finally, the speed of sound is:

$$
\rho c^{2}=\rho\left(\frac{\partial P}{\partial \rho}\right)_{s}=(\gamma-1)\left[\frac{\rho_{V} \rho_{L}}{\left(\rho_{L}-\rho_{V}\right)}\left(h_{V}-h_{L}\right)\right]
$$


Appendix C: convexity conditions for a mixture of stiffened gas

Starting from the relation (see Appendix A)

$$
\rho T d s=\frac{1}{\gamma-1} d P-\frac{c^{2}}{\gamma-1} d \rho
$$

And an expression of the differential of pressure:

$$
\frac{1}{\gamma-1} d P=d(\rho e)-\left(\frac{\partial \rho e}{\partial \rho}\right)_{P} d \rho
$$

And with the differential of $\rho e$ :

$$
d(\rho e)=\left(\frac{\partial \rho e}{\partial T}\right)_{\rho} d T+\left(\frac{\partial \rho e}{\partial \rho}\right)_{T} d \rho
$$

We can obtain the differential of temperature $d T$ :

$$
\left(\frac{\partial \rho e}{\partial T}\right)_{\rho} d T=\rho T d s-\left[\left(\frac{\partial \rho e}{\partial \rho}\right)_{T}-\left(\frac{\partial \rho e}{\partial \rho}\right)_{P}-\frac{c^{2}}{\gamma-1}\right] d \rho
$$

We deduce two equalities:

$$
\left(\frac{\partial T}{\partial s}\right)_{\rho}=\frac{\rho T}{\left(\frac{\partial \rho e}{\partial T}\right)_{\rho}} \quad ; \quad\left(\frac{\partial T}{\partial \rho}\right)_{s}=-\frac{\left(\frac{\partial \rho e}{\partial \rho}\right)_{T}-\left(\frac{\partial \rho e}{\partial \rho}\right)_{P}-\frac{c^{2}}{\gamma-1}}{\left(\frac{\partial \rho e}{\partial T}\right)_{\rho}}
$$

With the stiffened gas EOS, we have:

$$
\begin{aligned}
& \left(\frac{\partial \rho e}{\partial T}\right)_{\rho}=\frac{\rho C_{p}}{\gamma} \\
& \left(\frac{\partial \rho e}{\partial \rho}\right)_{T}=\frac{\gamma-1}{\gamma} \frac{\rho_{L}\left(e_{L}-q_{L}\right)-\rho_{V}\left(e_{V}-q_{V}\right)}{\rho_{L}-\rho_{V}}+\frac{1}{\gamma} \frac{\left(\rho_{L} C_{p_{L}}-\rho_{V} C_{p_{V}}\right) T}{\rho_{L}-\rho_{V}}+\rho_{V} \frac{q_{L}-q_{V}}{\rho_{L}-\rho_{V}}
\end{aligned}
$$

Finally the expression of dimensionless parameters:

$$
g=\frac{P}{\rho T^{2}}\left(\frac{\partial T}{\partial s}\right)_{\rho}=\frac{\gamma P}{\rho C_{p} T}
$$




$$
\Gamma=\frac{\rho}{T}\left(\frac{\partial T}{\partial \rho}\right)_{s}=\frac{\gamma}{\gamma-1} \frac{c^{2}}{C_{p} T}
$$




\section{References}

[1] Ahuja V, Hosangadi A, Arunajatesan S. Simulations of cavitating flows using hybrid unstructured meshes. Journal of Fluids Engineering 2001;123:331-340.

[2] Barberon T, Helluy P. Finite volume simulation of cavitating flows. Computers \& Fluids 2005;34:832-858.

[3] Barre S, Rolland J, Boitel G, Goncalves E, Fortes Patella R. Experiments and modelling of cavitating flows in Venturi: attached sheet cavitation. European Journal of Mechanics 2009, article in press.

[4] Chang CH, Liou MS. A robust and accurate approach to computing compressible multiphase flow: stratified flow model and AUSM+-up scheme. Journal of Computational Physics 2007;225:840-873.

[5] Chen HC, Patel VC. Near-Wall Turbulence Models for Complex Flows Including Separation. AIAA Journal 1985;26:641-648.

[6] Choi YH, Merkle CL. The application of preconditioning to viscous flows. Journal of Computational Physics 1993;105:207-223.

[7] Clerc S. Numerical simulation of the homogeneous equilibrium model for twophase flows. Journal of Computational Physics 2000;161:354-375.

[8] Coutier-Delgosha O, Fortes-Patella R, Reboud J-L. Simulation of unsteady cavitation with a two-equation turbulence model including compressibility effects. Journal of Turbulence 2002;3(58).

[9] Coutier-Delgosha O, Reboud J-L, Delannoy Y. Numerical simulation of the unsteady behaviour of cavitating flow. Int. Journal for Numerical Methods in Fluids 2003;42:527-548.

[10] Coutier-Delgosha O, Fortes Patella R, Reboud J-L, Hakimi N, Hirsch C. Numerical simulation of cavitating flow in 2D and 3D inducer geometries. Int. Journal for Numerical Methods in Fluids 2005;48:135-167. 
[11] Delannoy Y, Kueny J-L. Two phase flow approach in unsteady cavitation modelling. In: Cavitation and Multiphase Flow Forum, ASME-FED. 1990;98:153-158.

[12] De Vuyst F, Ghidaglia J-M, Le Coq G. On the numerical simulation of multiphase water flows with changes of phase and strong gradients using the Homogeneous Equilibrium Model. Int. Journal on Finite Volumes 2005;2(1):136.

[13] Edwards JR, Franklin RK. Low-Diffusion Flux Splitting methods for real fluid flows with phase transition. AIAA Journal 2000;38(9):1624-1633.

[14] Fortes Patella R, Barre S, Reboud J-L. Experiments and modelling of cavitating flows in Venturi : part II, unsteady cavitation. In: 6th International Symposium on Cavitation CAV2006, Wageningen, The Netherlands; September 2006.

[15] Gleize V, Costes M. Low-Mach-number preconditioning applied to turbulent helicopter fuselage flowfield computation. AIAA Journal 2003;41(4):653-662.

[16] Goncalves E, Houdeville R. Reassessment of the wall functions approach for RANS computations. Aerospace Science and Technology 2001;5:1-14.

[17] Guillard H, Viozat C. On the behaviour of upwind schemes in the low Mach number limit. Computers \& Fluids 1999;28:63-86.

[18] Harten A, Lax PD, Lervermore CD, Morokoff W. Convex entropies and hyperbolicity for general euler equations. SIAM Journal on Numerical Analysis 1998;35(6):2117-2127.

[19] Iben U, Wrona F, Munz C-D, Beck M. Cavitation in hydraulic tools based on thermodynamic properties of liquid and gas. Journal of Fluids Engineering 2002;124:1011-1017.

[20] Jameson A, Schmidt W, Turkel E. Numerical simulation of the Euler equations by finite volume method using Runge-Kutta time stepping schemes. AIAA Paper 81-1259; In: 14th Fluid and Plasma Dynamics Conference, Palo Alto, California; June 1981. 
[21] Jameson A. Time Dependent Calculations Using Mutligrid with Applications to Unsteady Flows past Airfoils and Wings. AIAA Paper 91-1259; In: 10th Computational Fluid Dynamics Conference, Honolulu, Hawai; June 1991.

[22] Kloczko T, Corre C, Beccantini A. Low-cost implicit schemes for all-speed flows on unstructured meshes. Int. Journal for Numerical Methods in Fluids 2008;58(5):493-526.

[23] Kunz RF, Boger DA, Stinebring DR, Chyczewski TS, Lindau JW, Gibeling HJ, Venkateswaran S, Govindan TR. A preconditioned Navier-Stokes method for two-phase flows with application to cavitation prediction. Computers \& Fluids 2000;29(8):849-875.

[24] Le Metayer O, Massoni J, Saurel R. Elaborating equations of state of a liquid and its vapor for two-phase flow models. Int. Journal of Thermal Sciences 2004;43:265-276.

[25] Lindau JW, Venkateswaran S, Kunz RF, Merkle CL. Development of a fully-compressible multi-phase Reynolds-Averaged Navier-Stokes model. AIAA Paper 2001-2648; In: 15th Computational Fluid Dynamics Conference, Anaheim, California; June 2001.

[26] Lindau JW, Kunz RF, Boger DA, Stinebring DR, Gibeling HJ. High Reynolds number, unsteady, multiphase CFD modeling of cavitating flows. Journal of Fluids Engineering 2002;124:124-607.

[27] Liu TG, Khoo BC, Xie WF. Isentropic one-fluid modelling of unsteady cavitating flow. Journal of Computational Physics 2004;201:80-108.

[28] Luo H, Baum JD, Lohner R. A fast, matrix-free implicit method for compressible flows on unstructured grids. Journal of Computational Physics 1998;146:664-690.

[29] Meng H, Yang V. A unified treatment of general fluid thermodynamics and its application to a preconditioning scheme. Journal of Computational Physics 2003;189:277-304. 
[30] Menter FR. Two-equation eddy-viscosity turbulence models for engineering applications. AIAA Journal 1994;32:1598-1605.

[31] Merci B, Steelant J, Vierendeels J, Riemslagh K, Dick E. Computational treatment of source terms in two-equation turbulence models. AIAA Journal 2000;38:2085-2093.

[32] Moreau JB, Simonin O, Habchi C. A numerical study of cavitation influence on diesel jet atomisation. In: 19th annual meeting of the institute for liquid atomization and spray systems, Notthingham, England; September 2004.

[33] Murrone A, Guillard H. A five equation reduced model for compressible two phase flows problems. Journal of Computational Physics 2005;202:664-698.

[34] Paillere H, Corre C, Garcia Cascales JR. On the extension of the AUSM+ scheme to compressible two-fluid models. Computers \& Fluids 2003;32:891-916.

[35] Pascarella C, Salvatore V, Ciucci A. Effects of speed of sound variation on unsteady cavitating flows by using a barotropic model. In: 5th International Symposium on Cavitation CAV2003, Osaka, Japon; November 2003.

[36] Perrier V. The Chapman-Jouguet closure for the Riemann problem with vaporization. SIAM Journal On Applied Mathematics 2008;68(5):1333-1359.

[37] Pouffary B, Fortes Patella R, Reboud J-L, Lambert P-A. Numerical Simulation of 3D Cavitating Flows: Analysis of Cavitation Head Drop in Turbomachinery. Journal of Fluids Engineering 2008;130(6),061301.

[38] Roe PL. Approximate Riemann solvers, parameters vectors, and difference schemes. Journal of Computational Physics 1981;43:357-372.

[39] Saurel R, Abgrall R. A simple method for compressible multifluid flows. SIAM Journal of Scientific Computing 1999;21(3):1115-1145.

[40] Saurel R, Cocchi J-P, Barry Butler P. Numerical study of cavitation in the wake of a hypervelocity underwater projectile. Journal of Propulsion and Power 1999;15(3):513-522. 
[41] Saurel R, Le Metayer O. A multiphase model for compressible flows with interfaces, shocks, detonation waves and cavitation. Journal of Fluid Mechanics 2001;431:239-271.

[42] Schmidt SJ, Sezal IH, Schnerr GH. Compressible simulation of high-speed hydrodynamics with phase change. In: European Conference on Computational Fluid Dynamics ECCOMAS 2006, Delft, The Netherlands; September 2006.

[43] Senocak I, Shyy W. A pressure-based method for turbulent cavitating flow computations. Journal of Computational Physics 2002;176:363-383.

[44] Shin BR, Iwata Y, Ikohagi T. Numerical simulation of unsteady cavitating flows using a homogeneous equilibrium model. Computational Mechanics 2003;30:388-395.

[45] Singhal AK, Athavale MM, Li H, Jiang Y. Mathematical basis and validation of the full cavitation model. Journal of Fluids Engineering 2002;124:617-624.

[46] Sinibaldi E, Beux F, Salvetti MV. A numerical method for 3D barotropic flows in turbomachinery. Flow Turbulence Combustion 2006 ;76:371-381.

[47] Song CS. Current status of CFD for cavitating flows. In: 9th International Symposium on Transport Phenomena and Dynamics of Rotating Machinery, Honolulu, Hawai; February 2002.

[48] Spalart PR, Allmaras SR. A one-equation turbulence model for aerodynamic flows. La Recherche Aerospatiale 1994;1:5-21.

[49] Tatsumi S, Martinelli L, Jameson A. Flux-Limited Schemes for the Compressible Navier-Stokes Equations. AIAA Journal 1995;33:252-261.

[50] Turkel E. Preconditioned methods for solving the incompressible and low speed compressible equations. Journal of Computational Physics 1987;172:277-298.

[51] Utturkar Y, Wu J, Wang G, Shyy W. Recent progress in modelling of cryogenic cavitation for liquid rocket propulsion. Progress in Aerospace Sciences 2005;41:558-608. 
[52] van Driest ER. On Turbulent Flow Near a Wall. Journal of Aeronautic Science 1957;23:1007-1011.

[53] Venkateswaran S, Lindau JW, Kunz RF, Merkle CL. Preconditioning algorithms for the computation of multi-phase mixture flows. AIAA Paper 20010279; In: $39^{\text {th }}$ Aerospace Sciences Meeting \& Exhibit, Reno, Nevada; January 2001.

[54] Ventikos Y, Tzabiras G. A numerical method for the simulation of steady and unsteady cavitating flows. Computers \& Fluids 2000;29:63-88.

[55] Viegas JR, Rubesin MW. Wall-Function Boundary Conditions in the Solution of The Navier-Stokes Equations for Complex Compressible Flows. AIAA Paper 831694; In: $16^{t} h$ Fluid and Plasma Dynamics Conference, Danver, Massachussetts; July 1983.

[56] Wallis G. One-dimensional two-phase flow. New York: McGraw-Hill; 1967.

[57] Wu J, Wang G, Shyy W. Time-dependent turbulent cavitating flow computations with interfacial transport and filter-based models. Int. Journal for Numerical Methods in Fluids 2005;49:739-761.

[58] Yeom G-S, Chang K-S. Numerical simulation of two-fluid two-phase flows by HLL scheme using an approximate jacobian matrix. Numerical Heat Transfer, Part B 2006;49:155-177. 
Table 1

Parameters of the stiffened gas EOS for cold water applied by different authors.

\begin{tabular}{llllll} 
authors & $\gamma$ & $P_{\infty}(\mathrm{Pa})$ & $\mathrm{q}(\mathrm{J} / \mathrm{kg})$ & $C_{p}(\mathrm{~J} / \mathrm{K} . \mathrm{kg})$ & $c(\mathrm{~m} / \mathrm{s})$ \\
\hline Saurel \& Abgrall [39] & 4.4 & $610^{8}$ & 0 & - & 1625 \\
Barberon\& Helluy [2] & 3 & $8.53310^{8}$ & $-0.114810^{7}$ & 4200 & 1569 \\
Paillere et al. [34] & 2.8 & $8.510^{8}$ & 0 & 4186 & 1486 \\
Le Metayer et al. [24] & 2.35 & $10^{9}$ & $-0.116710^{7}$ & 4268 & 1300 \\
Chang \& Liou [4] & 1.932 & $1.164510^{9}$ & 0 & 8095 & 1487 \\
\hline
\end{tabular}

Table 2

Parameters of the stiffened gas EOS for cold water.

\begin{tabular}{llllllll} 
& $\gamma$ & $P_{\infty}(\mathrm{Pa})$ & $\mathrm{q}(\mathrm{J} / \mathrm{kg})$ & $C_{p}(\mathrm{~J} / \mathrm{K} . \mathrm{kg})$ & $c(\mathrm{~m} / \mathrm{s})$ & $\rho_{\text {sat }}\left(\mathrm{kg} / \mathrm{m}^{3}\right)$ & $e_{\text {sat }}(\mathrm{J} / \mathrm{kg})$ \\
\hline liquid & 1.01 & $1.21110^{7}$ & $-0.114210^{7}$ & 4183 & 110.7 & 998.19 & 83779 \\
vapor & 1.32 & 0 & $0.198510^{7}$ & 1883 & 423 & 0.0173 & $2.40210^{6}$ \\
\hline
\end{tabular}

Table 3

Parameters of the barotropic law.

\begin{tabular}{lllll}
$A$ & $\rho_{L}^{\text {sat }}\left(\mathrm{kg} / \mathrm{m}^{3}\right)$ & $\rho_{V}^{\text {sat }}\left(\mathrm{kg} / \mathrm{m}^{3}\right)$ & $c_{\text {min }}(\mathrm{m} / \mathrm{s})$ & $\Delta P(\mathrm{~Pa})$ \\
\hline 0.999999 & 998.193 & 0.0173 & 0.857 & 576 \\
0.999999 & 998.16 & 0.0173 & 0.472 & 175 \\
\hline
\end{tabular}


Table 4

Parameters of the different steady computations.

\begin{tabular}{|c|c|c|c|c|c|}
\hline case & EOS & turb model & $c_{\min }(\mathrm{m} / \mathrm{s})$ & $\sigma_{\text {inlet }}$ & $L_{\text {cav }}(\mathrm{mm})$ \\
\hline 1 & $\mathrm{SG}$ & SA & 0.65 & 0.61 & 77.7 \\
\hline 2 & SG & SA & 0.65 & 0.608 & 79.8 \\
\hline 3 & $\mathrm{SG}$ & SA & 0.65 & 0.596 & 86.1 \\
\hline 4 & SG & KWSST & 0.65 & 0.61 & 70.1 \\
\hline 5 & $\mathrm{SG}$ & KWSST & 0.65 & 0.602 & 79.9 \\
\hline 6 & SG & KWSST & 0.65 & 0.595 & unstable \\
\hline 7 & baro & $\mathrm{SA}$ & 0.857 & 0.6087 & 80.2 \\
\hline 8 & baro & SA & 0.857 & 0.604 & 115.4 \\
\hline 9 & baro & SA & 0.857 & 0.592 & unstable \\
\hline 10 & baro & SA & 0.472 & 0.611 & 80.2 \\
\hline 11 & baro & SA & 0.472 & 0.605 & unstable \\
\hline 12 & baro & KWSST & 0.857 & 0.609 & 80.2 \\
\hline 13 & baro & KWSST & 0.857 & 0.604 & unstable \\
\hline 14 & baro & KWSST & 0.472 & 0.614 & 79.9 \\
\hline 15 & baro & KWSST & 0.472 & 0.608 & 103.8 \\
\hline 16 & baro & KWSST & 0.472 & 0.602 & unstable \\
\hline
\end{tabular}


Table 5

Parameters of the different unsteady computations.

\begin{tabular}{|c|c|c|c|c|c|}
\hline case & EOS & $c_{\min }(\mathrm{m} / \mathrm{s})$ & $\sigma_{\text {inlet }}$ & $L_{\text {cav }}(\mathrm{mm})$ & comments \\
\hline 1 & SG & 0.65 & 0.624 & $L=65.5 \mathrm{~mm}$ & stable sheet without re-entrant jet \\
\hline 2 & SG & 0.65 & 0.61 & $L=90 \mathrm{~mm}$ & stable sheet with small re-entrant jet \\
\hline 3 & SG & 0.65 & 0.593 & $L=95 \mathrm{~mm}$ & stable sheet with small re-entrant jet \\
\hline 4 & baro & 0.857 & 0.65 & $L=64 \mathrm{~mm}$ & quasi-stable sheet with re-entrant jet \\
\hline 5 & baro & 0.857 & 0.624 & $L=70 \mathrm{~mm}$ & quasi-stable sheet with re-entrant jet \\
\hline 6 & baro & 0.857 & 0.61 & $L=73 \mathrm{~mm}$ & quasi-stable sheet with re-entrant jet \\
\hline 7 & baro & 0.857 & 0.605 & $L \simeq 60 \mathrm{~mm}$ & break-off of the sheet \\
\hline 8 & baro & 0.472 & 0.645 & $L=54 \mathrm{~mm}$ & stable sheet with small re-entrant jet \\
\hline 9 & baro & 0.472 & 0.626 & $L=70 \mathrm{~mm}$ & quasi-stable sheet with re-entrant jet \\
\hline 10 & baro & 0.472 & 0.608 & $L=73 \mathrm{~mm}$ & quasi-stable sheet with re-entrant jet \\
\hline 11 & baro & 0.472 & 0.59 & $L \simeq 48 \mathrm{~mm}$ & break-off of the sheet \\
\hline
\end{tabular}




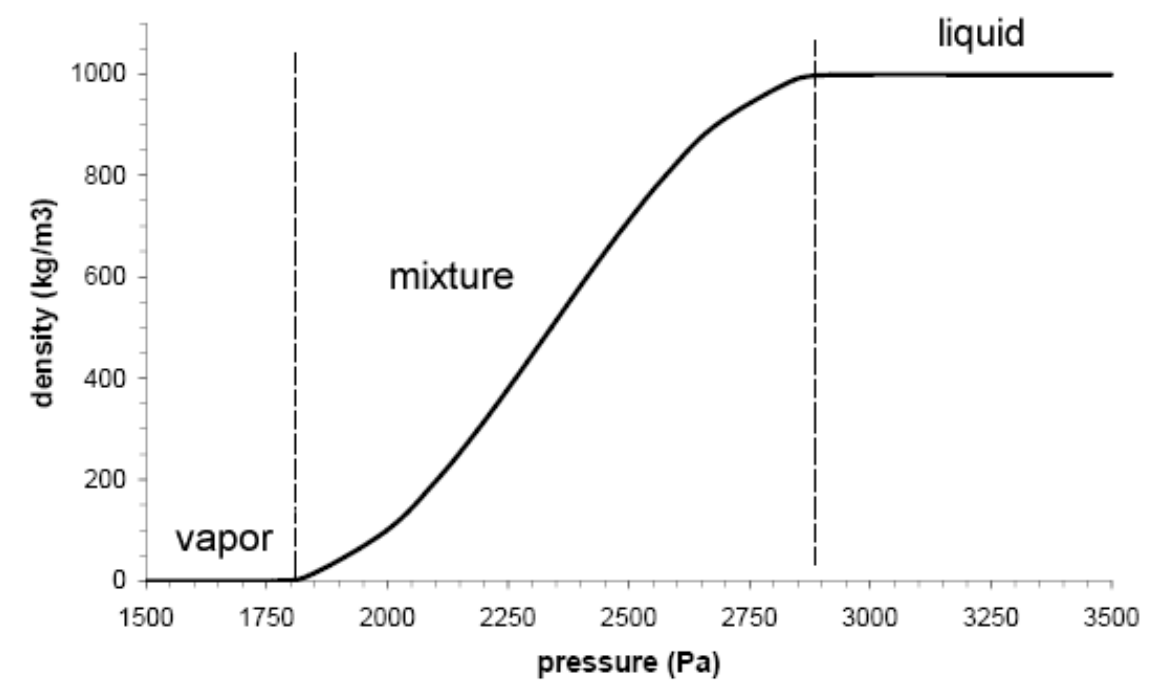

Fig. 1. The sinusoidal barotropic EOS.

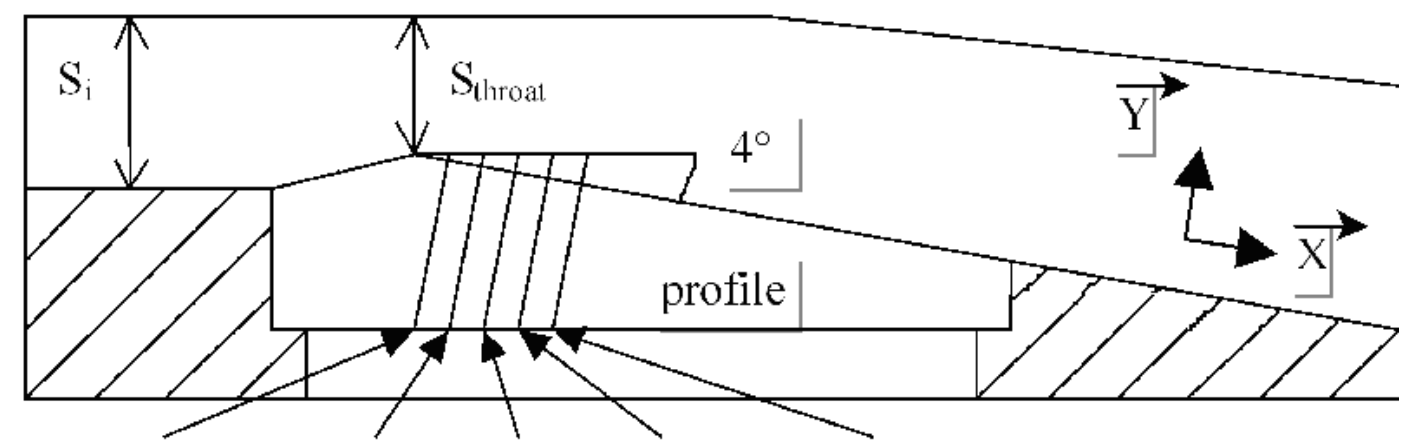

Station 1 Station 2 Station 3 Station 4 Station 5

Fig. 2. Schematic view of the Venturi profile.

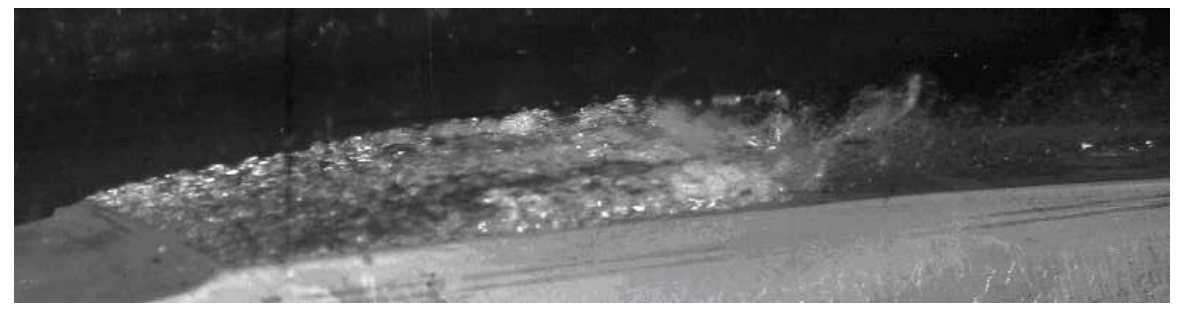

Fig. 3. Photograph of the cavity. 


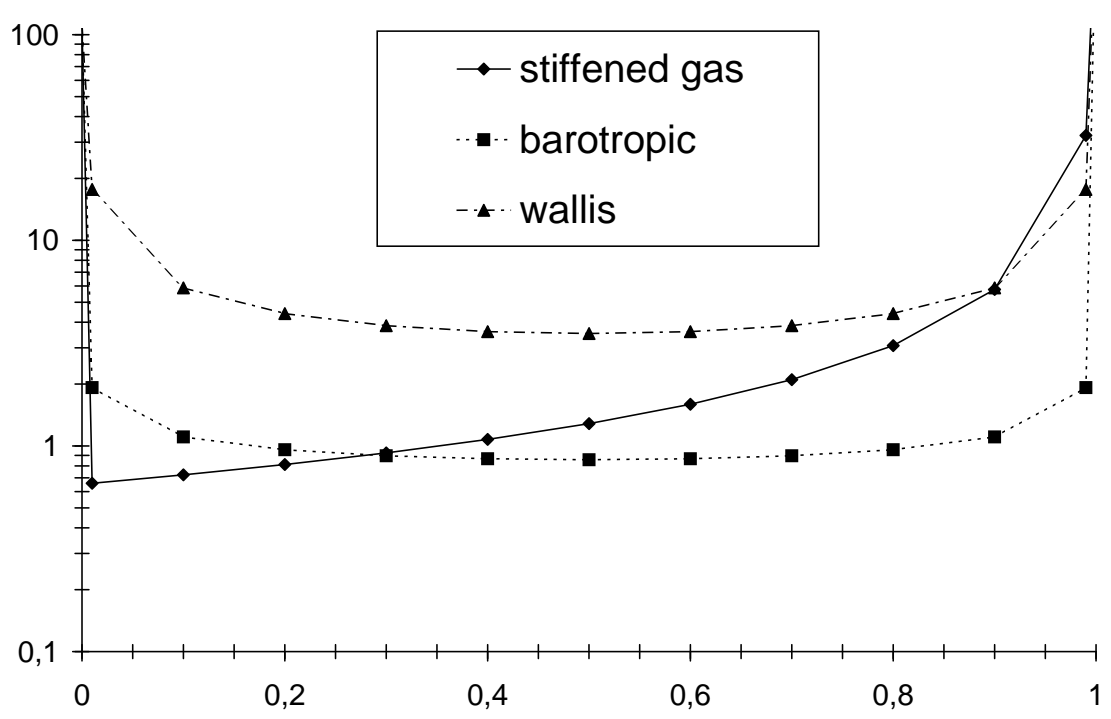

Fig. 4. Speed of sound for different models. 


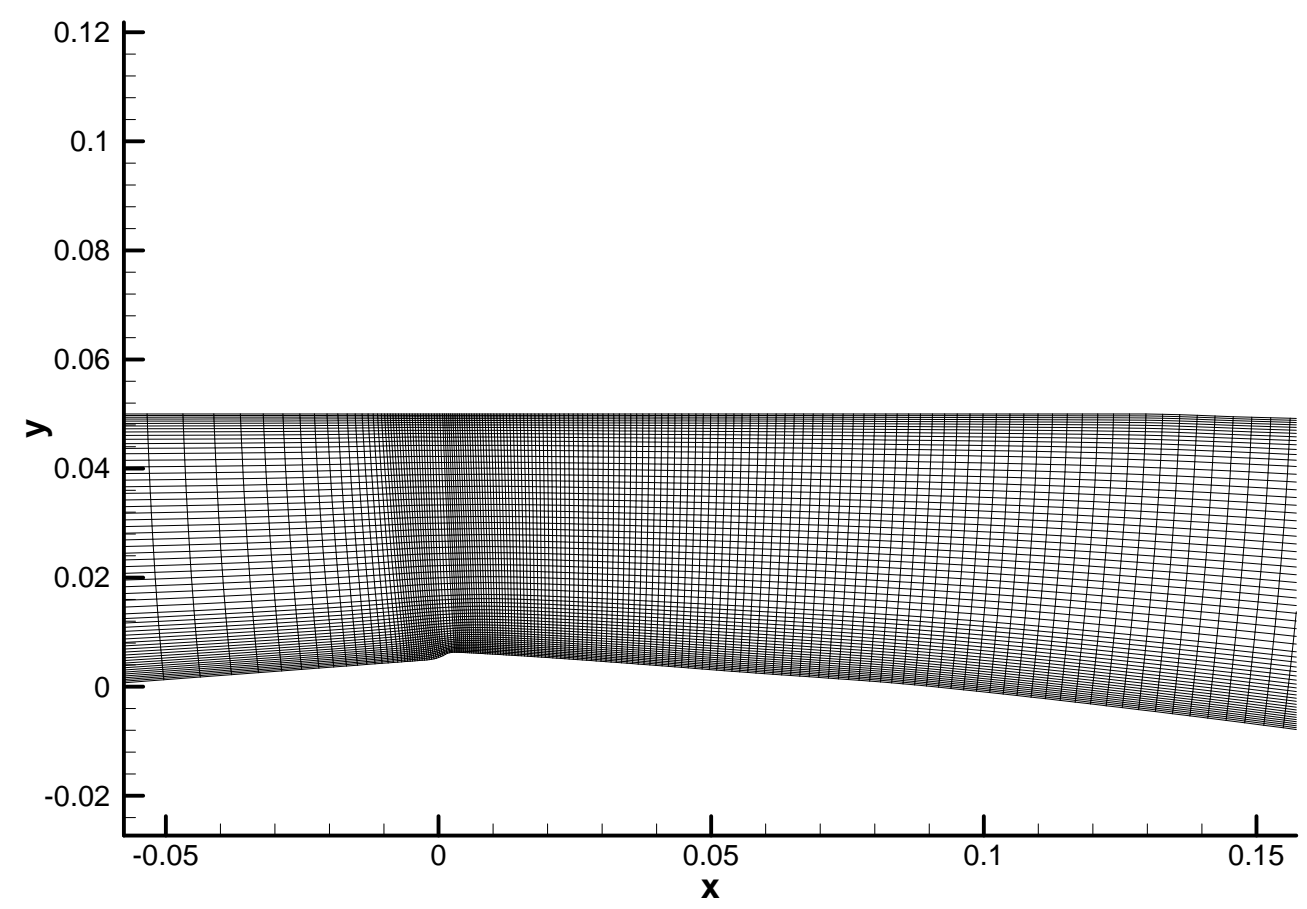

Fig. 5. Enlargement of the mesh near the Venturi throat. 

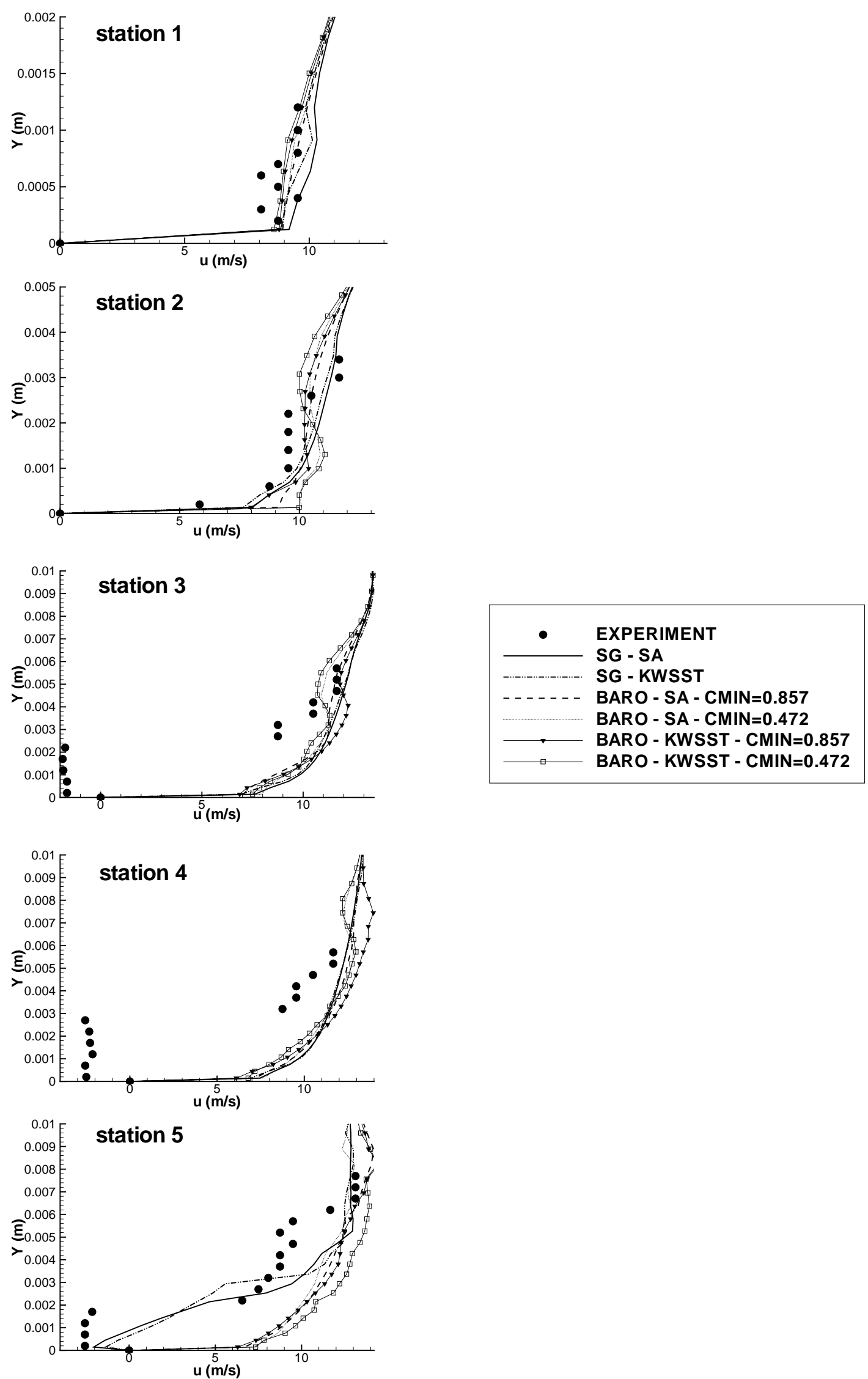

Fig. 6. Velocity profile from station 1 to 5 - steady computations. 

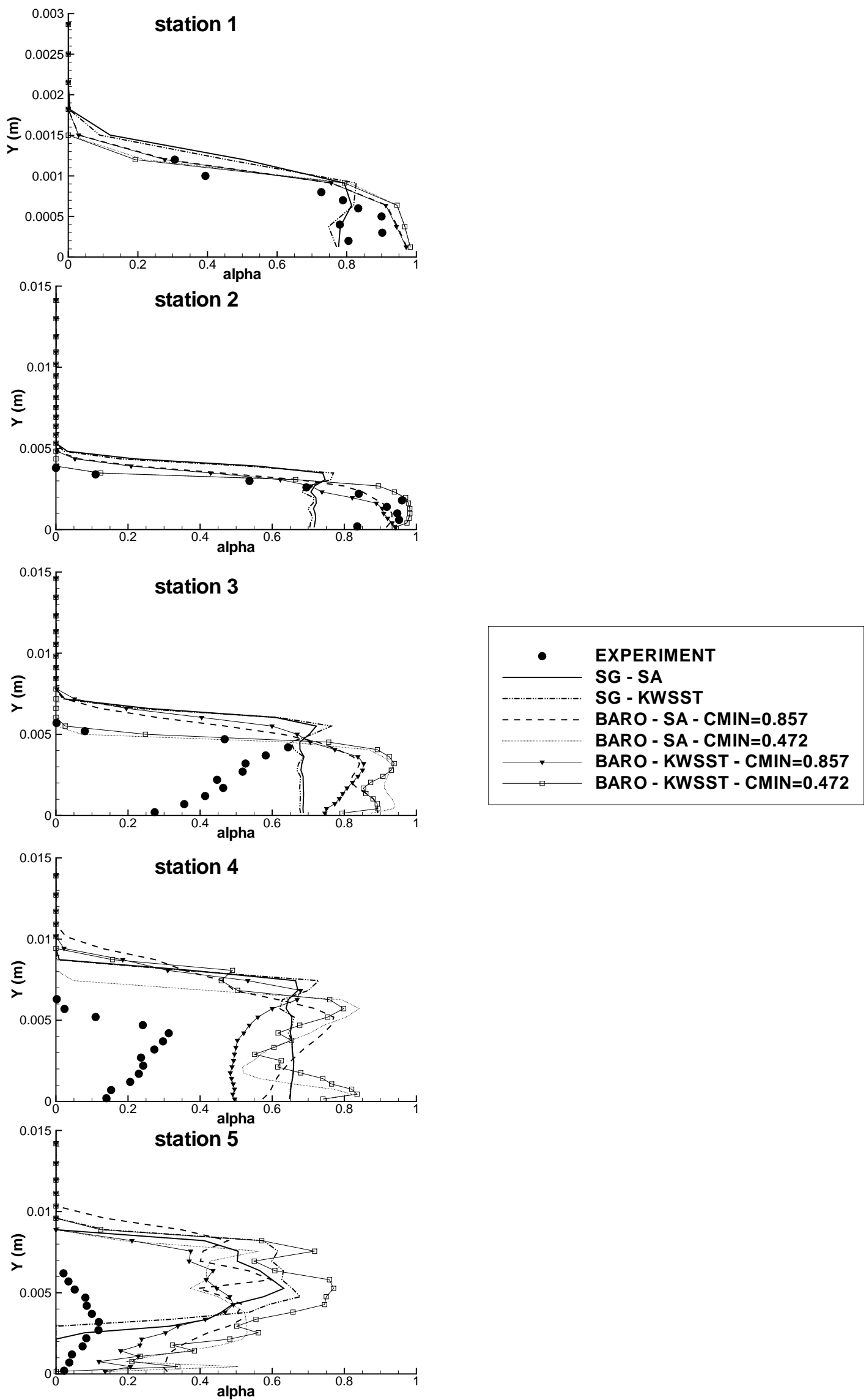

Fig. 7. Void ratio profile from station 1 to 5 - steady computations. 


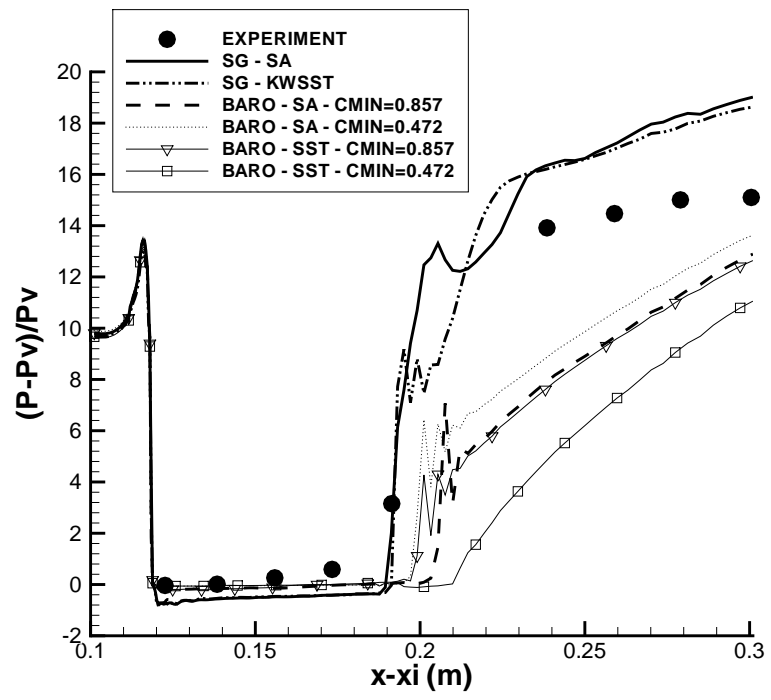

Fig. 8. Wall pressure evolution - steady computations. 

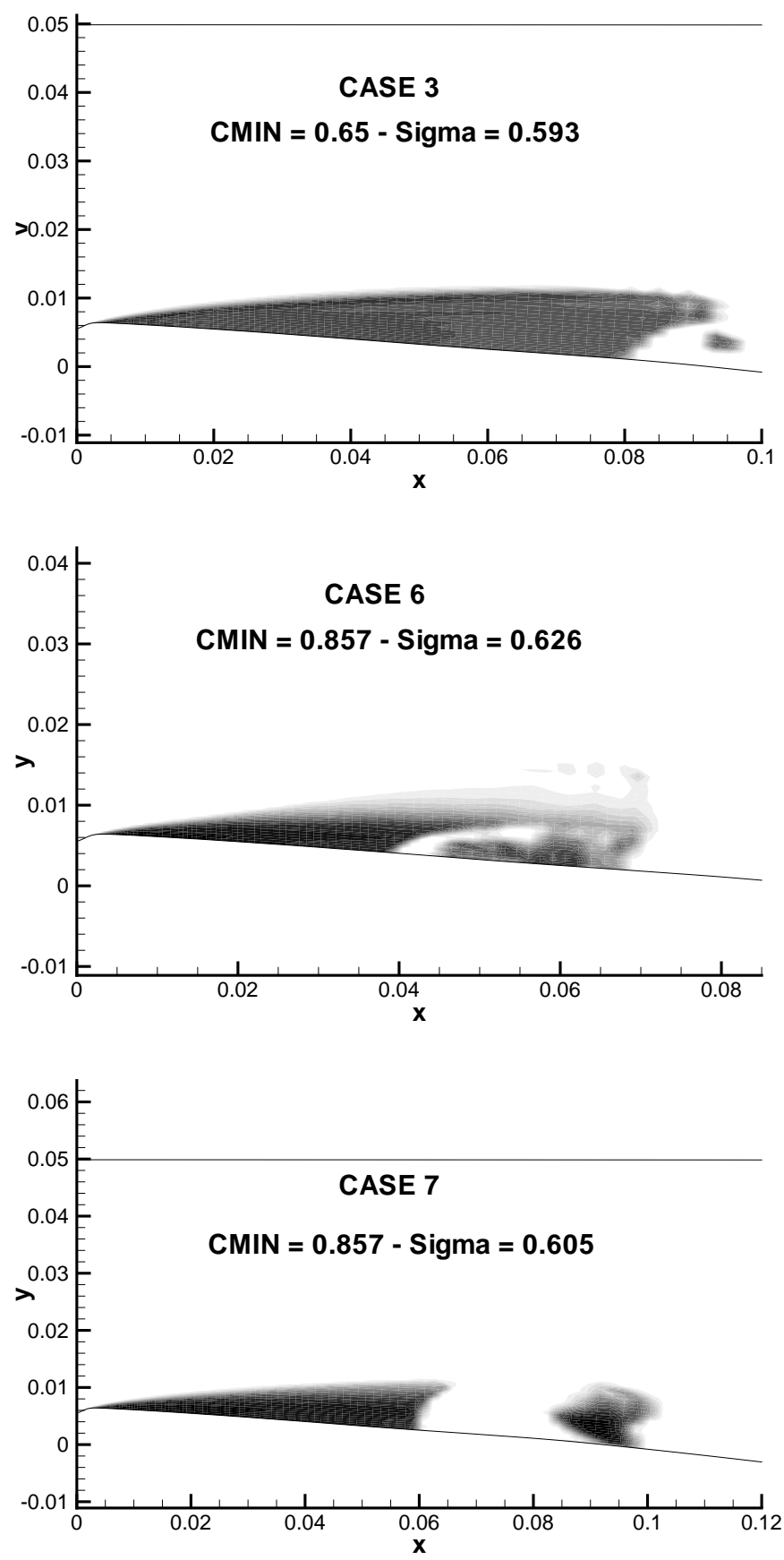

Fig. 9. The different behaviours of the cavity - Time-averaged void ratio. 

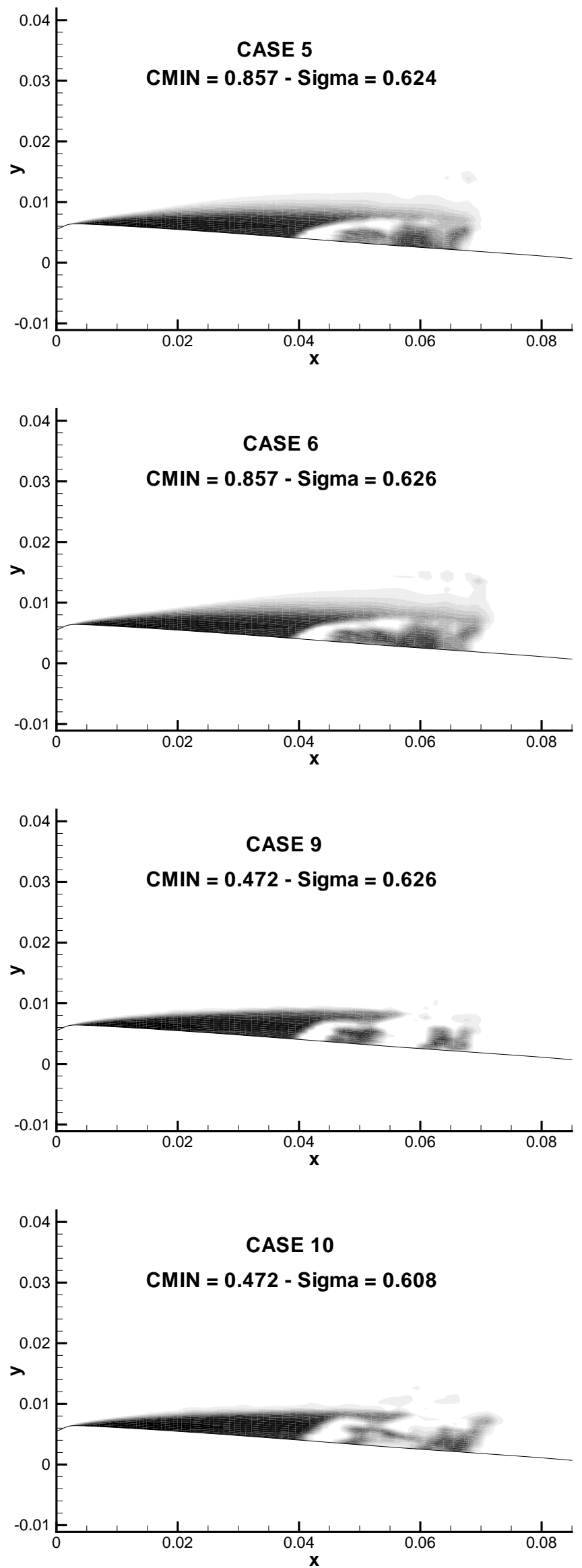

Fig. 10. Visualization of the cavity - Time-averaged void ratio. 

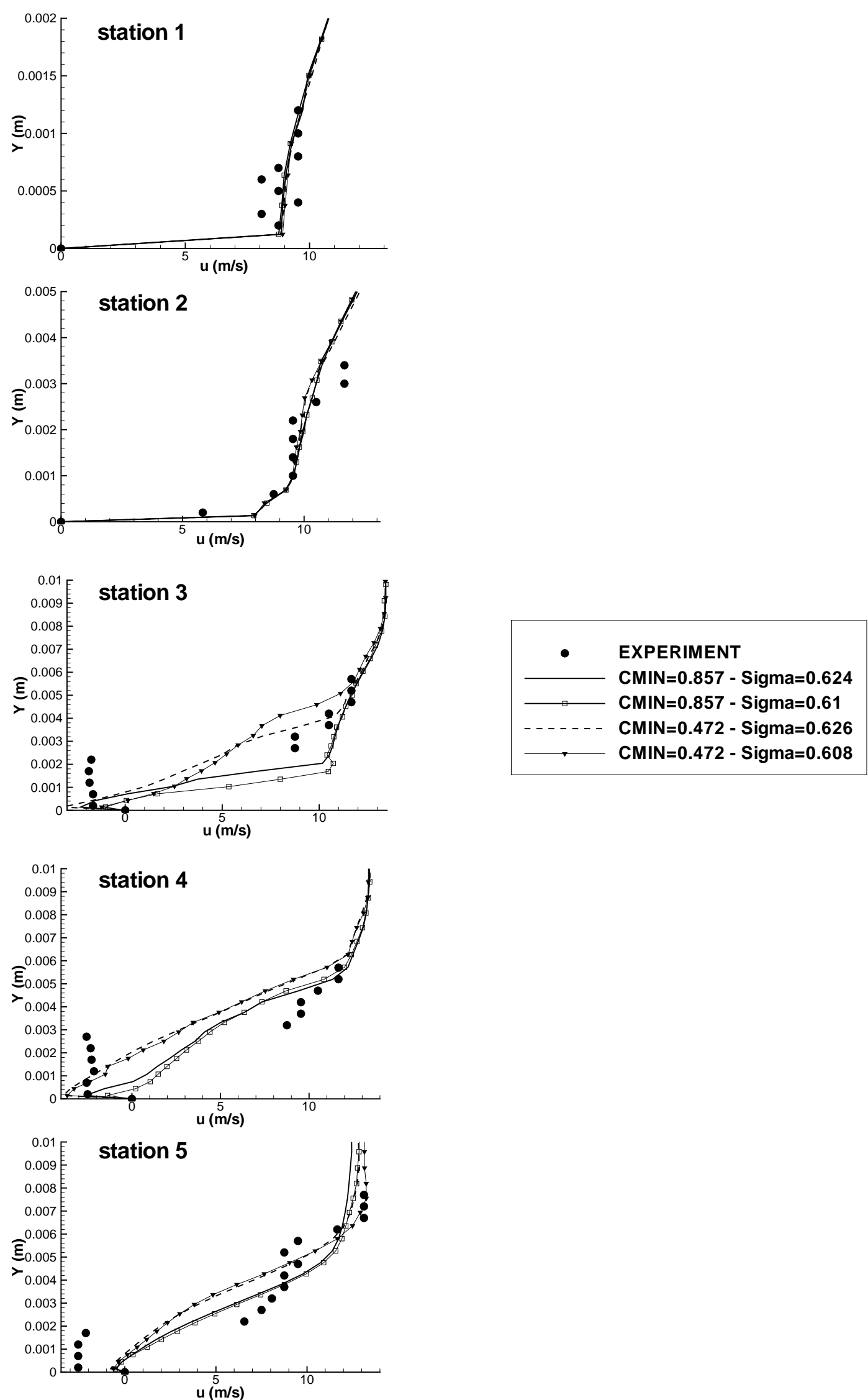

Fig. 11. Velocity profile from station 1 to 5 - unsteady computations. 

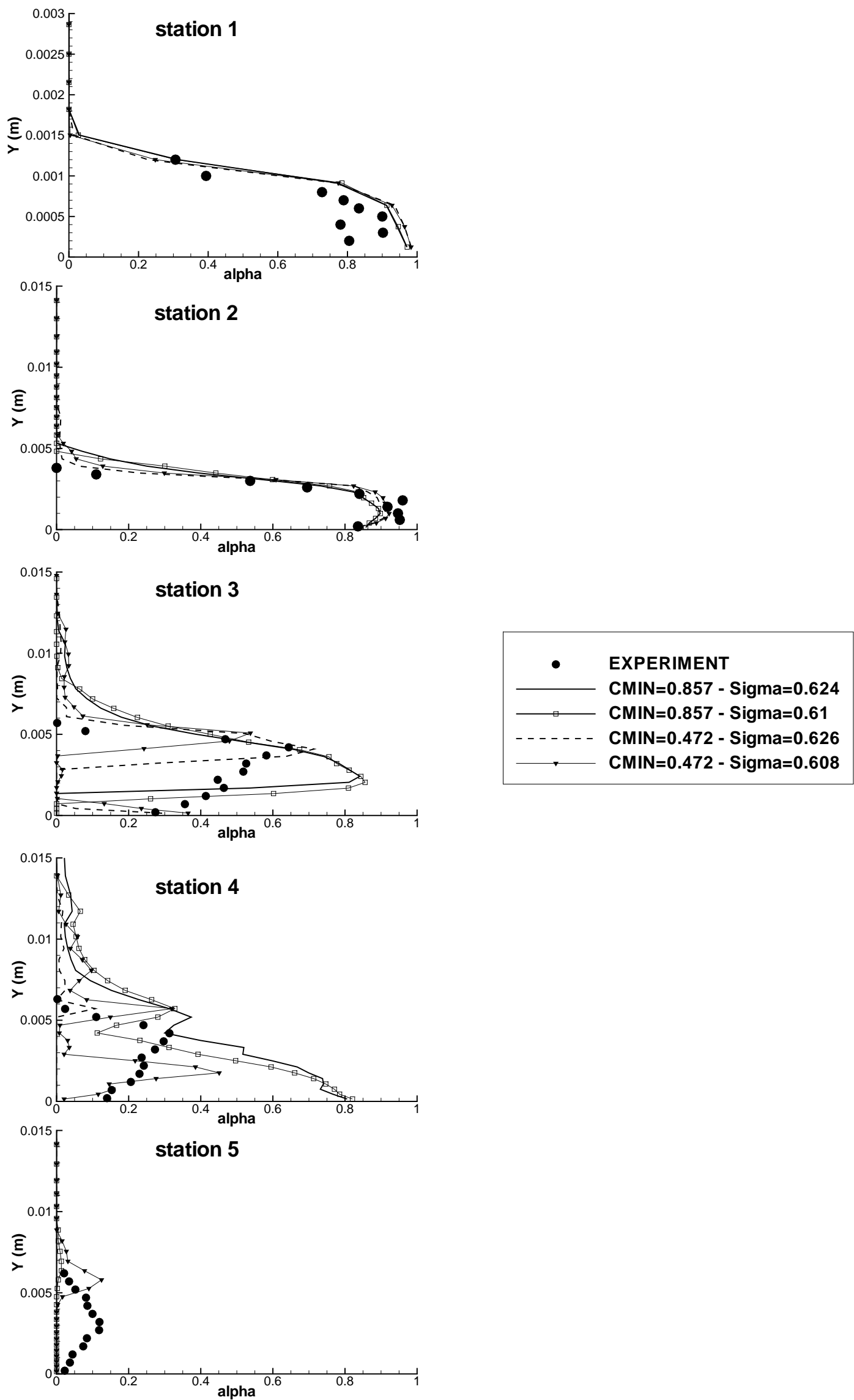

Fig. 12. Void ratio profile from station 1 to 5 - unsteady computations. 


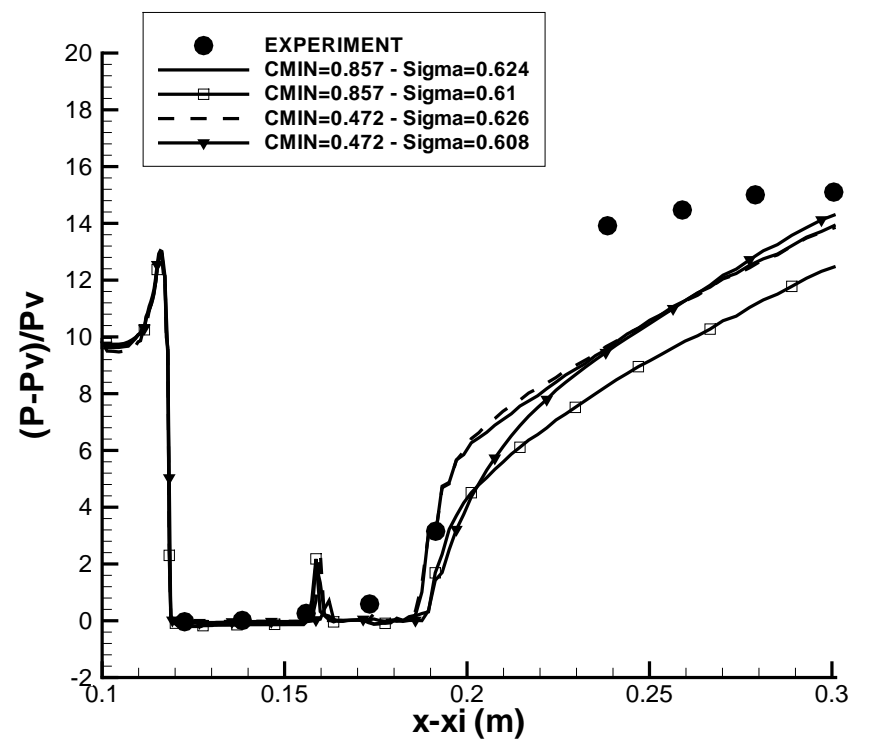

Fig. 13. Wall pressure evolution - unsteady computations. 